UUITP-08/00

USITP-00-15

hep-th/0009182

\title{
IIA/B, Wound and Wrapped
}

\author{
Ulf H. Danielsson, ${ }^{1}$ Alberto Güijosa, ${ }^{2}$ and Martín Kruczenski ${ }^{1}$ \\ ${ }^{1}$ Institutionen för Teoretisk Fysik, Box 803, SE-751 08 Uppsala, Sweden \\ ${ }^{2}$ Institute of Theoretical Physics, Box 6730, SE-113 85 Stockholm, Sweden \\ ulf@teorfys.uu.se, alberto@physto.se, \\ martin.kruczenski@teorfys.uu.se
}

\begin{abstract}
We examine the T-duality relation between $1+1$ NCOS and the DLCQ limit of type IIA string theory. We show that, as long as there is a compact dimension, one can meaningfully define an 'NCOS' limit of IIB/A string theory even in the absence of D-branes (and even if there is no $B$-field). This yields a theory of closed strings with strictly positive winding, which is T-dual to DLCQ IIA/B without any D-branes. We call this the Type IIB/A Wound String Theory. The existence of decoupled sectors can be seen directly from the energy spectrum, and mirrors that of the DLCQ theory. It becomes clear then that all of the different $p+1$ NCOS theories are simply different states of this single Wound IIA/B theory which contain D-branes. We study some of the properties of this theory. In particular, we show that upon toroidal compactification, Wound string theory is U-dual to various Wrapped Brane theories which contain OM theory and the ODp theories as special states.
\end{abstract}

September 2000 


\section{Introduction}

During the past couple of years there has been a lot of progress in the understanding of D-branes in the presence of magnetic fields and how these can be used to study Yang-Mills field theories with space/space non-commutativity [1], 2, 3]. Recently, as a generalization of this, the physics of D-branes with near-critical electric fields on their world-volumes has also attracted considerable attention [4, 5, 6]. Such theories have a peculiar space/time non-commutativity which in principle threatens their consistency [8, 9, 10]. However, as has been argued in the above cited works, the resulting theory is not a field theory, but a theory of open strings with a string scale of the same magnitude as the non-commutativity parameter. Contrary to the case of magnetic fields and space/space non-commutativity, then, the space/time noncommutativity and the stringiness cannot be disentangled from one another. The resulting theories are known as $(p+1)$-dimensional non-commutative open string theories (NCOS) [4, 5, 6]. The $3+1$ NCOS theory turns out to be S-dual to a theory with ordinary space/space non-commutativity, namely, 3+1 NCYM [5] (see also [11]).

Several successful calculations have been performed in NCOS theories. Examples include scattering amplitudes in 5, 12, 13, and supergravity duals and finite temperature physics in [14, 15, 16] (see also [17]-26]). NCOS theories have the usual rules for computing scattering amplitudes, except for the appearance of Moyal phases which depend on the ordering of the open string vertex operators [ [4, 5, 12], and are thus the source of non-commutativity. The closed string sector can be seen to decouple leaving a theory which contains only open strings [4, [], unless, as Klebanov and Maldacena have pointed out [12], one compactifies the theory on a circle along the direction of the electric field. One then finds that closed strings with strictly positive winding number have a finite energy in the NCOS limit, and interact in a non-trivial way with the brane.

It has also been noted in [1, 12, 18] that upon compactification along the direction of the electric field NCOS theories are T-dual to the discrete light-cone quantization (DLCQ) of Type II theories. DLCQ of M-theory and string theory has been the subject of intensive research in the Matrix theory context [27, 28]. As explained by Seiberg [29] (see also [30]), DLCQ can be defined by considering the theory on a spatial circle of vanishing size and then boosting along the direction of the circle to get a (finite-size) almost lightlike circle. The T-duality between the IIA/B NCOStheory and the DLCQ of IIB/A arises because a D $p$-brane with a near-critical electric field T-dualizes to a $\mathrm{D}(p-1)$-brane moving at the speed close to that of light [12, 4 . This is, in fact, precisely the condition for the $\mathrm{D}(p-1)$-brane to remain in the DLCQ spectrum after the boost: if the D-brane were instead at rest it would acquire an infinite energy. A more detailed exploration of this T-duality relation between the NCOS and DLCQ limits is the central subject of the present paper.

We begin in Section 2 with a brief review of the NCOS limit, focusing for concreteness on the $(1+1)$-dimensional case. We then proceed to exhibit the explicit

\footnotetext{
${ }^{1}$ See [7] for a discussion of theories with lightlike non-commutativity.
} 
mapping between the NCOS and DLCQ descriptions. In the process, we encounter a small puzzle. Starting with a brane carrying an electric field, T-duality will transform the electric field into a velocity. By performing a boost one can then put the brane at rest. The worldsheet fields will then obey the usual Neumann/Dirichlet boundary conditions, and one would not expect any Moyal phases associated with non-commutativity even in the DLCQ limit - the T-dual image of the NCOS limit. The resolution of this is to remember that T-duality requires compactification on a circle, and converts momentum into winding number. The left- and right-moving momenta are then different. It will be shown in Section 8 that combining this with the fact that the circle is boosted, one indeed reproduces the expected Moyal phases, even though they now have a different origin.

A related puzzle is the following. As explained in [12], the closed string spectrum of the compactified $1+1$ NCOS theory is the one which results from the presence of a near-critical $B_{01}$-field. As can be seen from the formulas in that paper, such a $B$-field in fact modifies the mass-shell condition for closed strings in a way that is very similar to the light-cone energy-momentum relation. At first sight this is somewhat confusing, since the $B$-field can in this case (as opposed to the usual case of a $B_{i j}$-field on a two-torus) be gauged away from the bulk and into the D-brane, where it cannot have any effect on the closed string spectrum. As we will discuss in detail in Section 3, the key point is that the $B$-field is merely an artifact that plays the same role as the boost in the Seiberg procedure [29, 30]. A boost does not change the physics, but in the case of [29, 30] maps the variables to simpler ones. In particular, it subtracts an infinite contribution from the energy.

It follows from this observation that it should be possible to deduce the decoupling of closed strings with non-positive winding directly in the gauge where it is $F_{01}$ and not $B_{01}$ that becomes critical, and in Section 3 we verify that this is indeed the case. It becomes apparent then that this decoupling has nothing to do with the presence of the D-branes or background fields, and that, as long as there is a compact direction, one can meaningfully define an 'NCOS' limit of the full IIA/B string theory (with or without branes). We study the resulting ten-dimensional theory in Section \&, and we discover that its defining property is the fact that all objects in it must carry strictly positive F-string winding. It is thus natural to call this the (IIA or IIB) Wound String theory. It is T-dual to DLCQ (IIB or IIA) string theory, and the corresponding decoupling arguments are mirror images of one another. The various $p+1$ NCOS theories are then different states in this single unifying theory - namely, those states that contain a $\mathrm{D} p$-brane wrapping the compact direction. We emphasize that the Wound theory is well-defined and non-trivial even in the absence of such branes.

In Section 5 we examine the Wound IIA/B theories compactified on transverse tori. Through diverse dualities, we are driven to define various Wrapped $p$-brane theories which are the natural generalizations of the Wound string idea: these theories are obtained as limits of string/M-theory with (at least) $p$ compact directions, and all objects in them must carry strictly positive $p$-brane wrapping number on this $p$ - 
torus. Just like the various NCOS theories are different classes of states in a single Wound string theory, the recently discovered OM theory [6, 31] (see also [32]) and OD $p$ theories [33, 6] are specific classes of states in a broader framework: as it will be shown in Section 5, OM theory is the Wrapped M2-brane theory in the presence of M5-branes which wrap the 'Wrapped' directions, whereas each of the OD $p$ theories is understood to be the Wrapped Dp-brane theory in the presence of NS5-branes. Section 5 includes a discussion of the relation of these theories to the known S-duals for NCOS theories [5, 6, 34] and the theories encountered in Seiberg's derivation of the Matrix description for DLCQ IIA/M-theory [29, 30].

We conclude in Section 6, where we in particular emphasize the distinction between the Matrix and Wrapped points of view.

\section{$21+1$ NCOS vs. DLCQ IIA}

\subsection{Review of the NCOS limit}

In this section we will review the NCOS limit defined in [4, 5, 6] specializing to the $(1+1)$-dimensional case; the higher-dimensional cases will be discussed in Section 5 . Consider then an $(N, 1)$ string, i.e., a bound state of a D-string and $N$ fundamental strings. We take the string to lie along the $x^{1}$ direction, and the background (string frame) metric to be flat and diagonal,

$$
g_{a b}=\eta_{a b}, \quad g_{i j}=h \delta_{i j}, \quad a, b=0,1, \quad i, j=2, \ldots, 9 .
$$

As indicated, we have split the ten spacetime directions into those parallel and perpendicular to the string, $\mu=(a, i)$.

The $N$ fundamental strings in the bound state are represented as $N$ units of electric flux. The relation between the electric field and $N$ follows from the Dirac-Born-Infeld action (see, e.g., 35),

$$
\frac{2 \pi \alpha^{\prime} F_{01}}{\sqrt{1-\left(2 \pi \alpha^{\prime} F_{01}\right)^{2}}}=N g_{s} \Longrightarrow \quad E=\frac{N g_{s}}{\sqrt{\left(N g_{s}\right)^{2}+1}}
$$

where $E \equiv 2 \pi \alpha^{\prime} F_{01}$ is the electric field measured in units of its critical value.

The net effect of the electric field on open string dynamics can be summarized by introducing the effective string metric, coupling constant, and non-commutativity parameter [3, 4]

$$
G_{a b}=\left(1-E^{2}\right) \eta_{a b}, \quad G_{i j}=h \delta_{i j} ; \quad G_{o}^{2}=g_{s} \sqrt{1-E^{2}} ; \quad \theta=2 \pi \alpha^{\prime} \frac{E}{1-E^{2}} .
$$

Let $E=1-\epsilon / 2$. The non-commutative open string (NCOS) limit [4, 5, 6] is a near-critical limit

$$
\epsilon \rightarrow 0, \quad \text { with } \quad \alpha^{\prime}=\alpha_{e}^{\prime} \epsilon \rightarrow 0, \quad h=\epsilon \rightarrow 0 ; \quad \alpha_{e}^{\prime}, N \quad \text { fixed }
$$


Notice that an effective Regge slope, $\alpha_{e}^{\prime}$, has been introduced; it is this slope which is held fixed in the limit.

It follows from (2) and (3) that in the NCOS limit?

$$
\begin{aligned}
g_{s} & =\frac{1}{N \sqrt{\epsilon}} \rightarrow \infty, \\
G_{o}^{2} & =\frac{1}{N}, \\
G_{\mu \nu} & =\epsilon \eta_{\mu \nu}, \\
\theta & =2 \pi \alpha_{e}^{\prime} .
\end{aligned}
$$

The end result is a string theory of open strings with tension set by $\alpha_{e}^{\prime}$, coupling constant $G_{o}^{2}$, and non-commuting $x^{0}, x^{1}$ directions, $\left[x^{0}, x^{1}\right] \sim i \theta$ [ [4, 5, 6]. The scaling of the parameter $h$ in the transverse closed string metric (1) expresses the rescaling of distances which is needed for the limit to produce a full-fledged string theory. As one approaches criticality, the strings in effect become tensionless only in the direction of the electric field, so one must zoom-in on a small transverse region (or equivalently, scale down the longitudinal direction) in order for the transverse oscillations of the strings to remain in the theory. Were it not for this rescaling, the strings would behave as rigid rods. Notice also that the fact that $G_{\mu \nu}$ in (7) vanishes as $\epsilon \rightarrow 0$ is compensated in the open-string mass-shell condition by the rescaling of $\alpha^{\prime}$, so the metric of the NCOS theory is in effect $\eta_{\mu \nu}$.

Next, we compactify the theory along the direction of the electric field (and the string), identifying $x^{1} \simeq x^{1}+2 \pi R$, and regarding $R$ as fixed in the NCOS limit. It has been shown in [12 that wound closed strings are then present in the spectrum of the theory (these become infinitely massive in the infinite volume limit, of course). The intuitive picture is as follows. The near-critical electric field forces the ends of the open strings to move away from each other along the positive $x^{1}$ direction. In the non-compact case, it would thus be energetically forbidden (in the strict NCOS limit) for the ends to join together to form a closed string. If the $x^{1}$ direction is compact, on the other hand, the ends can meet after having moved around the circle a certain number of times, thus giving rise to closed strings with strictly positive winding number [12], $w>0$.

Despite this intuitive picture, it is surprising that the presence of the electric field could have an effect on the closed strings, since they are supposed to be electrically neutral. Klebanov and Maldacena [12] circumvented this difficulty by gauging the electric field $E$ on the brane into a spacetime $B$-field - it is then easy to see the change in the closed string energy spectrum as $B$ approaches criticality. In Section 3 we will discuss this in detail, showing that the gauge transformation that shifts $E$ into $B$ is T-dual to a boost. We will also explain how the decoupling of closed strings with $w \leq 0$ can be seen directly in the $(E \neq 0, B=0)$ gauge, and from this

\footnotetext{
${ }^{2}$ The near-critical limit for the $(N, 1)$ string, but taking $g_{s}$ fixed and $N$ large, as opposed to $N$ fixed and $g_{s}$ large, was analyzed in $[17,36$, 1 . The physics of the two limits is in some respects similar.
} 
it will become clear that the same decoupling will take place in the absence of any D-branes - even if there is no $B$-field!

\subsection{Longitudinal T-duality and Matrix String Theory}

We are now interested in studying the effect of T-duality on the NCOS theory. It was pointed out in [4, 12, 18] that this should yield the DLCQ IIA string theory. Here we try to make this notion more precise. For this we will describe the theory in terms of the parent IIB theory, with a single D1-brane and a near-critical electric field $E=1-\epsilon / 2, \epsilon \rightarrow 0$. T-dualizing along the compact direction, we obtain an equivalent description of the system in terms of a D0-brane moving with speed close to that of light,

$$
v=\partial_{0} X^{1}=1-\epsilon / 2,
$$

in a IIA theory whose parameters follow from the rules of T-duality:

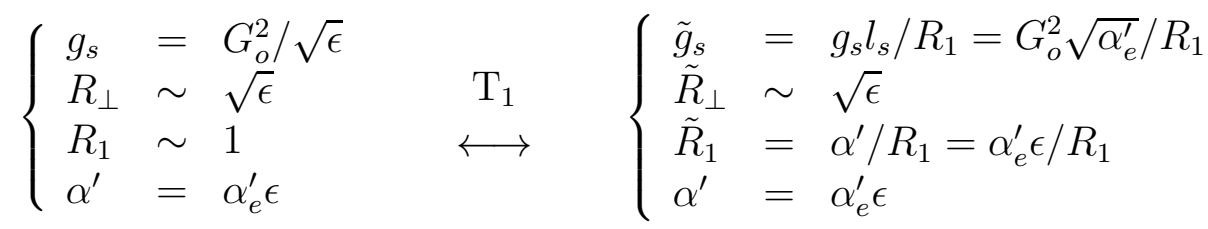

Here $R_{\perp}$ denotes any (proper) length in the transverse (non-compact) directions. The scaling $R_{\perp} \sim \sqrt{\epsilon}$ results from the transverse metric (1). The $N$ units of fundamental string winding on the D1-brane become $N$ units of Kaluza-Klein momentum for the D0-brane, $P_{1}=N / \tilde{R} \rightarrow \infty$.

The scaling in the right-hand side of (10) is precisely the one that defines the DLCQ limit of IIA string theory, in the sense of [29, 30]. Following Seiberg, we rescale all lengths in the theory by a factor of $\sqrt{\epsilon}$, to work in units in which the string length is finite,

$$
\tilde{l_{s}}=\sqrt{\alpha_{e}^{\prime}}
$$

The change of units makes all quantities finite except $\tilde{R}_{1}$, which becomes $\tilde{R}_{1}=$ $\sqrt{\epsilon} \alpha_{e}^{\prime} / R_{1}$. We next carry out a large boost along direction 1 , with velocity $\beta=1-\epsilon / 2$, to a frame $\mathrm{F}^{\prime}$ where the circle lies (almost) along $x^{\prime-} \equiv\left(x^{\prime 0}-x^{1}\right) / 2$ and has a finite radius

$$
\tilde{R}_{-}=\frac{\tilde{R}}{\epsilon}=\frac{\alpha_{e}^{\prime}}{R_{1}} .
$$

We have thus shown explicitly that $(1+1)$-dimensional NCOS with parameters $G_{o}^{2}=1 / N, \alpha_{e}^{\prime}$, compactified on a circle of radius $R$, is T-dual to DLCQ IIA string

\footnotetext{
${ }^{3}$ Boosting the system by an additional finite amount just rescales this null radius. We have chosen the boost parameter to equal the velocity (9), so the D0-brane will in fact be at rest along the $x^{\prime 1}$ direction in the frame $\mathrm{F}^{\prime}$. There is a free parameter describing the 'finite portion' of the boost, and (12) reflects our specific choice for this parameter, which translates into a specific relation between the NCOS and DLCQ reference frames $\mathrm{F}$ and $\mathrm{F}^{\prime}$.
} 
theory with coupling constant

$$
\tilde{g}_{s}=G_{o}^{2} \frac{\sqrt{\alpha_{e}^{\prime}}}{R_{1}}
$$

string length (11), and null radius (12), in the presence of a single D0-brane, and with total longitudinal momentum $P_{-}^{\prime}=N / \tilde{R}_{-}$. Notice that (11), (12) and (13) are in fact just the naive T-duality relations, but they use $\alpha_{e}^{\prime}$ instead of $\alpha^{\prime}$, and relate a spatial compactification to a lightlike one. This relation evidently extends to the case with an arbitrary number, $K$, of D1-branes: $1+1 \operatorname{NCOS}(K)$ with parameters $G_{o}^{2}=K / N, \alpha_{e}^{\prime}$, on a circle of radius $R$, is T-dual to DLCQ IIA with parameters $\tilde{g}_{s}=G_{o}^{2} \sqrt{\alpha_{e}^{\prime}} / R$ and (11), on a circle of radius (12), in the presence of $K$ D0-branes, and with $N$ units of longitudinal momentum.

Notice that we are using the term 'T-duality' in a slightly extended sense, since the mapping that relates the two theories is the composition of ordinary T-duality, a change of units, and a boost. The change of units can of course be avoided by presenting the NCOS theory directly in the new units: this would entail keeping $l_{s}$ and the transverse metric parameter $h$ fixed, while scaling the parallel components of the metric according to $g_{a b}=\epsilon^{-1} \eta_{a b}$. As shown in [4], this results in an NCOS theory with parameters $G_{o}^{2}=1 / N, \alpha_{e}^{\prime}=\alpha^{\prime}, \theta=2 \pi \alpha^{\prime}, G_{\mu \nu}=\eta_{\mu \nu}$. In Section 3.2 we will see that the boost can also be avoided, by working in the gauge where it is $B$ and not $E$ that becomes critical.

We note in passing that the above DLCQ IIA string theory (after the change of units) can be equivalently formulated as M-theory with eleven-dimensional Planck length

$$
\tilde{l}_{P}=\tilde{g}_{s}^{1 / 3} \tilde{l}_{s}=\left(\frac{\alpha_{e}^{\prime 2}}{N R}\right)^{1 / 3},
$$

compactified on a null circle of radius (12), and a transverse spatial circle of radius

$$
\tilde{R}_{10}=\tilde{g}_{s} \tilde{l}_{s}=\frac{\alpha_{e}^{\prime}}{N R}
$$

with $N$ units of longitudinal momentum, and one unit of transverse momentum.

DLCQ IIA string theory is believed to admit a non-perturbative description in terms of Matrix string theory (i.e., $\mathcal{N}=8$ super-Yang-Mills in $1+1$ dimensions) [37, 38, 39, and the series of steps we have followed above is in fact exactly the reverse of the sequence of dualities used to justify [29] the Matrix string theory conjecture. The situation is summarized in Fig. 1.

The S-duality between $1+1$ NCOS and $1+1 U(N)$ SYM with $K$ units of electric flux indicated in Fig. 1 has been scrutinized in [6, 12, 13]. As explained in those works, the SYM coupling is related to the NCOS parameters through $g_{Y M}^{2}=1 /\left(2 \pi \alpha_{e}^{\prime} G_{o}^{4}\right)$. The effective dimensionless coupling at energy $E$ is therefore $g_{\text {eff }}^{2} \simeq 1 /\left(\alpha_{e}^{\prime} G_{o}^{4} E^{2}\right)$. At low energies the theory is strongly coupled, but the presence of the compact circle

\footnotetext{
${ }^{4}$ It is easy to see that this is in agreement with the known relation to DLCQ IIA parameters [39], $g_{Y M}^{2}=\tilde{R}_{-}^{2} /\left(2 \pi \tilde{g}_{s}^{2} \tilde{l}_{s}^{4}\right)$.
} 


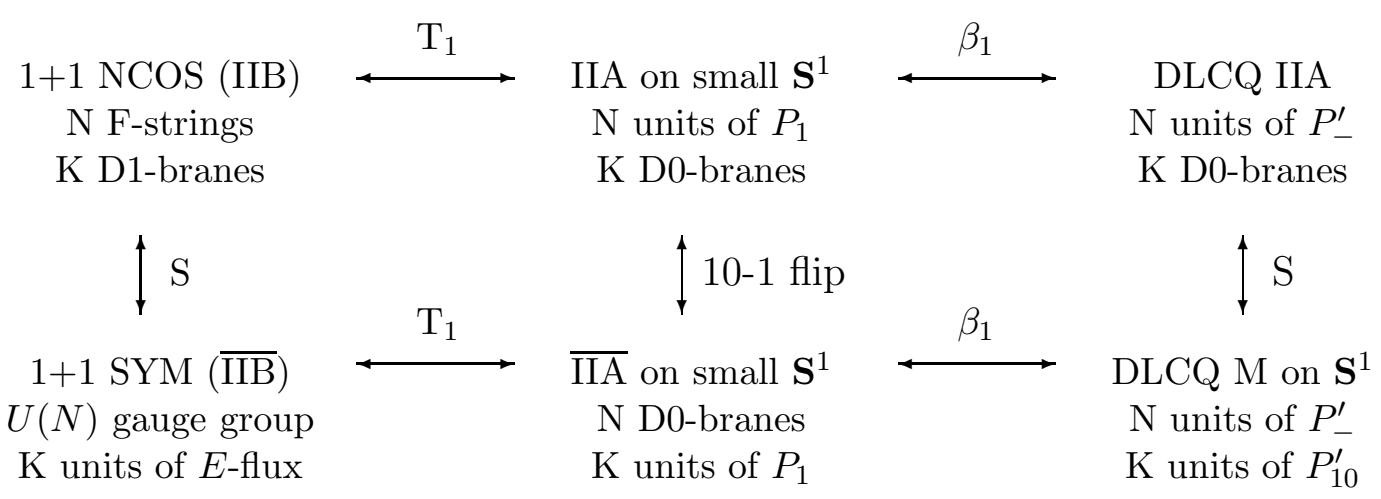

Figure 1: The $1+1$ NCOS/DLCQ IIA duality web. Seiberg's derivation of a nonperturbative Matrix formulation for DLCQ IIA (DLCQ M-theory on a transverse $\mathbf{S}^{1}$ ) proceeds along the top (bottom) line and then down to arrive at $1+1$ SYM. $\beta_{1}$ denotes a boost along $x^{1}$.

provides an infrared cutoff $1 / R$, meaning that $g_{\text {eff }}^{2} \leq R^{2} /\left(\alpha_{e}^{\prime} G_{o}^{4}\right)$. In Section 4 we will find that this is indeed the inverse of the effective coupling of the NCOS theory, which can be argued to be $\sqrt{\alpha_{e}^{\prime}} G_{o}^{2} / R$.

It is interesting to work out the complete $1+1$ NCOS $\leftrightarrow$ DLCQ IIA dictionary, which follows from the usual rules of T-duality. The NCOS open strings are dual to the usual open strings ending on (and describing the dynamics of) the IIA D0-brane. NCOS and IIA closed strings are mapped onto one another, with their winding and Kaluza-Klein numbers interchanged. (We have already seen one example of this: the $N$ fundamental strings - i.e., units of electric flux - appearing in the definition of the NCOS theory correspond to the $N$ units of longitudinal IIA momentum, $P_{-}^{\prime}=N / \tilde{R}_{-}$.) The fact that NCOS winding is necessarily positive [12], $w>0$, is therefore equivalent to the well-known fact that in DLCQ longitudinal momentum is strictly positive, $p_{-}^{\prime}>0$. The $x^{-}$winding number in the DLCQ theory is of course arbitrary, just like the dual NCOS Kaluza-Klein number. It is worth emphasizing that the standard interchange of momentum and winding has in this case the rather non-standard effect of mapping a theory with gravity to a non-gravitational theory. Of course, in our postMatrix/Maldacena era [27, 40], the equivalence of a non-gravitational theory and a theory of gravity is slightly less surprising.

Klebanov and Maldacena [12] noted that it is possible for the 'NCOS D-string' to emit wound closed strings into the bulk: in the parent IIB theory, this just corresponds to the $(N, 1)$ bound state dissociating into an $(N-w, 1)$ bound state and a number of fundamental strings with winding numbers $w_{A}>0$ such that $\sum_{A} w_{A}=w$. In the S-dual IIB description, one has instead a $(1, N)$ string out of which some Dstrings are separated. In SYM language, this is expressed by the breaking $S U(N) \rightarrow$ $S U(N-w) \times U(1)^{w}$, keeping the unit of $E$-flux in the $S U(N-w)$ part. Now we see that in the T-dual DLCQ IIA language, this corresponds to the process in which a D0-brane with $N$ units of longitudinal momentum emits closed strings into the bulk with $p_{(A)-}^{\prime}>0$. The cost in light-cone energy that this process entails can easily be 
seen to agree with the one discussed in [12] for the NCOS theory. The identification between a $S U\left(N^{\prime}\right)$ subsector of Matrix string theory carrying $K$ units of $E$-flux and $K$ DLCQ IIA D0-branes with total longitudinal momentum $p^{\prime}=N^{\prime} / \tilde{R}_{-}$was discussed in 39.

One of the most surprising aspects of the $1+1$ NCOS theory is the decoupling of the massless open string modes, inferred in [12 from the identification of these modes with the free $U(1)$ sector of the $1+1 U(N)$ SYM theory, and verified through explicit computation of scattering amplitudes with massless open string vertices. In the Matrix literature it is well-known that the $U(1)$ part of of the SYM Lagrangian describes the center-of-mass motion of the DLCQ IIA system in the transverse directions [27, 39], whose decoupling is obvious. Notice, however, that massless open string vertices in NCOS are associated only with quantum fluctuations of the center-of-mass dynamics of the D1-brane (and the $N$ adsorbed F-strings), which under T-duality becomes the IIA D0-brane. When the NCOS or DLCQ IIA state under consideration includes additional closed strings, these give rise to a separate contribution to the center-of-mass modes. Since this contribution is certainly incorporated in the free $U(1)$ factor of $1+1 \mathrm{SYM}$, there is a question as to what is it that decouples in NCOS amplitudes which include closed string vertices. It would be interesting to have a closer look at this point.

From the T-dual perspective it is clear that the presence of the D1-brane in the NCOS theory is not essential: in the DLCQ IIA theory it is just as interesting to consider states without any D-branes. Similarly, it is natural to ask what DLCQ IIA states with other D-branes correspond to in the NCOS language. We will elaborate on these issues in Sections 3 and 4 .

To complete the dictionary, we should also understand how the non-commutativity manifests itself in the T-dual picture. The defining property of NCOS theories is the fact that scattering amplitudes are computed with the usual rules but including Moyal phases when two operators are interchanged. In the T-dual description one has a D0-brane in motion, which is brought to rest by means of the (large) boost. The boundary conditions for the world-sheet fields will then be the usual ones (Neumann in direction 0 and Dirichlet in the rest). The propagator $\left\langle X^{\mu} X^{\nu}\right\rangle$ will clearly not give rise to a phase in this case.

To see what happens consider two open strings with momentum $n^{(1)} / R_{1}$ and $n^{(2)} / R_{1}$ in NCOS prior to taking the limit. These states T-dualize into open strings ending on the D0-brane with winding number $n^{(1)}$ and $n^{(2)}$. This calculations are standard (see, e.g., 41]). Due to the winding number one has different left and right momenta,

$$
p_{\mu L, R}=\left(p_{0}, \pm \frac{n \tilde{R}_{1}}{\alpha^{\prime}}, p_{\perp}\right)
$$

After the boost the D0-brane is at rest but the circle is no longer purely spatial. The momenta respecting the new periodicity are of the form

$$
p_{\mu L, R}=\left(\gamma\left(p_{0} \mp \beta \frac{n \tilde{R}_{1}}{\alpha^{\prime}}\right), \gamma\left( \pm \frac{n \tilde{R}_{1}}{\alpha^{\prime}}-\beta p_{0}\right), p_{\perp}\right)
$$




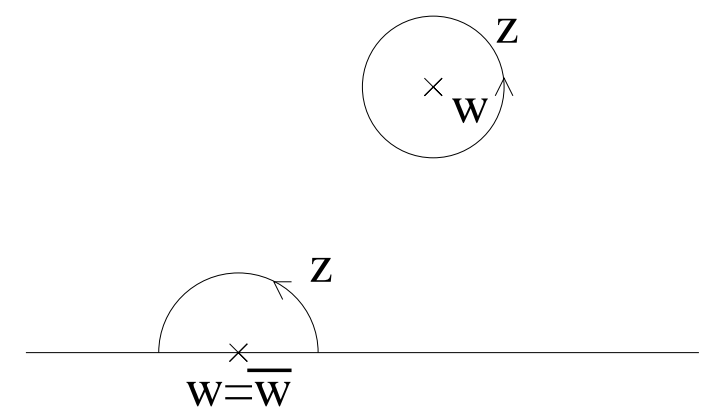

Figure 2: A closed string vertex encircling another, and the analogous operation for open string vertices, which inverts their order. In the latter case there is an extra phase due to the fact that $\left\langle X_{\mu}(z) \tilde{X}_{\nu}(\bar{w})\right\rangle \neq 0$.

where $\beta=1-\epsilon / 2$ is the original speed of the D0-brane and $\gamma=1 / \sqrt{1-\beta^{2}}$. The propagators have the standard form

$$
\begin{aligned}
\left\langle X_{\mu}(z) X_{\nu}(w)\right\rangle & =-\alpha^{\prime} \eta_{\mu \nu} \ln (z-w) \\
\left\langle X_{\mu}(z) \tilde{X}_{\nu}(\bar{w})\right\rangle & =\alpha^{\prime} \delta_{\mu \nu} \ln (z-\bar{w})
\end{aligned}
$$

where we took into account that $X_{0}$ obey Neumann boundary conditions and the rest Dirichlet. If one takes a closed string vertex of momentum $p^{(1)}$ around another with momentum $p^{(2)}$ there is a phase change $\exp \left(2 \pi i\left(p_{L}^{(1)} p_{L}^{(2)}-p_{R}^{(1)} p_{R}^{(2)}\right)\right.$, which for consistency must equal unity. However, if one does the same for open string vertices, as indicated in Fig. 2, there is an extra phase coming from the fact that $z$ is also going (half-way) around $\bar{w}$ (and not only around $w$ as in the closed string case) extra phase is given by

$$
\exp \left(i \pi \alpha^{\prime}\left(p_{L}^{(1)} p_{R}^{(2)}-p_{R}^{(1)} p_{L}^{(1)}\right)\right)=\exp \left(4 \pi i \beta \gamma^{2} \tilde{R}_{1}\left(p_{0}^{(1)} n^{(2)}-p_{0}^{(2)} n^{(1)}\right)\right.
$$

Taking the $\epsilon \rightarrow 0$ limit and using the T-duality relations one finds that this phase is finite and given by

$$
\exp \left(2 \pi i \alpha_{e}^{\prime}\left(p_{0}^{(1)} p_{1}^{(2)}-p_{1}^{(2)} p_{0}^{(2)}\right)\right)
$$

with $p^{(1,2)}=n^{(1,2)} / R_{1}$. This last expression is the expected Moyal phase, with noncommutativity parameter $\theta^{01}=2 \pi \alpha_{e}^{\prime}$.

In 112 a general argument for the vanishing of NCOS amplitudes with at least one massless open string vertex was presented, based on the observation that in that case the propagator is in effect an analytic function, so the integral over the position of the vertex on the boundary can be pulled to the interior and shrunk to zero size.

\footnotetext{
${ }^{5}$ Here we consider open string vertices to be functions of $X(z), \tilde{X}(\bar{z})$ with $X(z)-\tilde{X}(\bar{z})$ contractions removed. Equivalently, one can use closed string vertices which are close to the boundary, and then the same result follows using OPE's to reduce to open string vertices.
} 
From (17) we see that the T-dual statement is that for a massless particle $p_{L}=0$, which means the vertex is a purely analytic function (since $p_{L}$ is the momentum multiplying $\tilde{X}(\bar{z})$ ) and then the same reasoning follows. This of course will be valid in the presence of handles, since the circle can be pulled past them; but the argument might fail if one inserts a closed string vertex operator, for example.

In the Matrix program, DLCQ is not an end in itself: the ultimate goal is to provide a description of the corresponding decompactified theory. This is an enormously difficult task, since it involves understanding the large $N$ limit of the model in question. So it is natural to wonder whether the duality to NCOS theory could afford any new insight into the nature of this limit. A glance at (6) is encouraging: $N \rightarrow \infty$ is the NCOS weak-coupling limit, $G_{o}^{2} \rightarrow 0$ ! Upon closer examination, however, the situation is not as fortunate: one must remember that in the decompactification limit, the longitudinal momentum $P_{-}^{\prime}=N / \tilde{R}_{-}$(for a state with no winding) should be held fixed and finite, and through (12) this translates into the requirement that the NCOS radius $R$ scale as the inverse of $N$. This is not surprising: as we have seen, the NCOS description is related by T-duality to the IIA theory whose decompactified limit we are trying to understand. The NCOS description is not particularly transparent in this limit.

In the Matrix context, it has been argued that the decompactified IIA theory can also be obtained from a 'DLCQ' limit in which $N \propto \epsilon^{-1} \rightarrow \infty$ as $\epsilon \rightarrow 0$, while the radius of the spatial circle in the (infinite momentum) frame $\mathrm{F}$ is held fixed in string units [42, 43. In that case the circle in frame $\mathrm{F}^{\prime}$ has growing radius, $\tilde{R}_{-} \propto \epsilon^{-1}$, and is not strictly lightlike. After T-duality (and a change of units), one arrives at a IIB description with $l_{s} \propto \sqrt{\epsilon}$ and arbitrary coupling constant $g_{s}$. If $K$ D0branes are present in the IIA picture, the $K$ corresponding D1-branes share $N \propto \epsilon^{-1}$ units of $E$-flux. The radius $R$ of direction $x^{1}$ is again fixed in string units. This limit, studied in [36, 17], is distinct from the NCOS limit, although they share some features. The point we are making is that it must coincide with the large $N$ limit of the NCOS description. That this is surprising is perhaps more easily appreciated in the S-dual IIB description: we are saying that, as $N \rightarrow \infty$, the physics of a system of $N$ D1-branes becomes independent of the string coupling. Evidence for this type of independence (in a different context) was given in [42, 43]. This property is to some extent responsible for the success of the Matrix model, and makes it clear that the large $N$ limit is highly non-trivial.

We should also remark that, whereas Matrix string theory is conjectured to provide a complete non-perturbative (second-quantized) description of DLCQ IIA string theory, the S-dual $(1+1)$ NCOS picture discussed above utilizes just the usual perturbative rules of the first-quantized framework. The same is of course true if one attempts to 'directly' describe the DLCQ IIA theory, along the lines of 44, 45, 46, 47.

\section{Closed Strings, $B$-fields, and Boosts}




\subsection{The Closed String Spectrum}

As mentioned in the previous section, Klebanov and Maldacena [12 discovered that upon compactification, the $1+1$ NCOS theory contains closed strings with strictly positive winding number. The authors of 112 determined the spectrum of NCOS closed strings by working in the gauge where $B_{01}$ (and not $E$ ) becomes critical. Let us review their argument. The mass formula for closed strings in a $B$-field [41, 12] is

$$
\left(p_{0}\right)^{2}+2 p_{0} B_{01} \frac{w R}{\alpha^{\prime}}-\left(\frac{w R}{\alpha^{\prime}}\right)^{2}\left(1-B_{01}^{2}\right)-\left(\frac{n}{R}\right)^{2}-p_{\perp}^{2}-\frac{2}{\alpha^{\prime}}\left(N_{L}+N_{R}\right)=0
$$

supplemented by the level-matching condition

$$
N_{L}-N_{R}=n w .
$$

Interpreting (23) as a mass formula is actually quite confusing: the nine-dimensional mass depends on the energy $p_{0}$ (and therefore on $p_{\perp}$ ). Notice that this is true even of the ten-dimensional mass. It is clear that this is a purely stringy phenomenon, given that it only affects states with non-zero winding, $w \neq 0$. The best way to view (23) is as a quadratic equation for the energy. From it one finds the two solutions

$$
p_{0}=-\frac{B_{01} w R}{\alpha^{\prime}} \pm \sqrt{\left(\frac{w R}{\alpha^{\prime}}\right)^{2}+\left(\frac{n}{R}\right)^{2}+p_{\perp}^{2}+\frac{2}{\alpha^{\prime}}\left(N_{L}+N_{R}\right)} .
$$

Notice that the quadratic term in $B_{01}$ has canceled inside the square-root. If we restrict attention to the physical range $\left|B_{01}\right| \leq 1$, it is clear that we must choose the upper sign in (25) to have $p_{0}>0$.

Let us now examine how (25) behaves in the NCOS limit. Writing $B_{01}=1-\epsilon / 2$, $\alpha^{\prime}=\alpha_{e}^{\prime} \epsilon$ and $p_{\perp}^{2}=k_{\perp}^{2} / \epsilon$, the NCOS limit is $\epsilon \rightarrow 0$ holding $\alpha_{e}^{\prime}$ and $k_{\perp}$ fixed. For $w=0$, it is easy to see from (25) that $p_{0} \propto \epsilon^{-1 / 2} \rightarrow \infty$, (unless $k_{\perp}=N_{L}=N_{R}=0$ ). For $w \neq 0$, the NCOS limit turns (25) into

$$
p_{0}=\frac{R}{\epsilon \alpha_{e}^{\prime}}(-w+|w|)+\frac{w R}{2 \alpha_{e}^{\prime}}+\frac{\alpha_{e}^{\prime}}{2|w| R} k_{\perp}^{2}+\frac{N_{L}+N_{R}}{|w| R}+\mathcal{O}(\epsilon) .
$$

Clearly the energy diverges unless we arrange for the first term to drop out, i.e., if $w>0$. In that case we find

$$
p_{0}=\frac{w R}{2 \alpha_{e}^{\prime}}+\frac{\alpha_{e}^{\prime}}{2 w R} k_{\perp}^{2}+\frac{N_{L}+N_{R}}{w R},
$$

which indeed agrees with the result of [12] (obtained by noting that in the NCOS limit (23) turns into a linear equation - this is true only if one assumes that $p_{0}$ is finite in the limit). A whole tower of closed string excitations is thus seen to be present in the NCOS theory. These states show up as poles in NCOS open string scattering amplitudes [12].

\footnotetext{
${ }^{6}$ This restriction is clear if there are D-branes present: for $\left|B_{01}\right|>1$ there are tachyonic modes in the open string spectrum. In the absence of D-branes (i.e., in a theory with closed strings only), one can observe that $\left|B_{01}\right|>1$ is excluded since either choice of sign for the square root would lead to states with negative energy. After T-dualizing it will be even easier to understand this restrictionsee below.
} 


\section{$3.2 \quad B$ is for Boost}

While the approach of Klebanov and Maldacena [12] reviewed in the previous subsection yields the correct spectrum (i.e., the one seen in open string scattering), it is somewhat mysterious that the observed decoupling appears to depend on the presence of the $B$-field, or in other words, on the choice of a particular gauge. In the gauge where $B=0$ and it is $E$ that becomes critical, the (free) closed string spectrum must be the standard one, because closed strings are electrically neutral. In this subsection and the next we will clarify this issue.

We start from the following observation. It is well-known that a $\mathrm{D} p$-brane with a constant electric field $E$ is equivalent a $\mathrm{D}(p-1)$-brane moving at speed $v=E$ : the two are mapped onto one another under T-duality along the direction of the field. It should therefore be possible to transform away a constant electric field through a T-duality transformation followed by an appropriate boost and a new T-duality. Since we also know that a gauge transformation can exchange an electric field for a $B_{01}$-field, we conclude that the effect of the latter field on a closed string can be understood by boosting a system with $B=0$.

Let us now fill in the details of the above argument. We begin with the IIB theory with $x^{1}$ compactified on a circle of radius $R$ and with $B=0$. After a simple T-duality exchanging the winding and momentum, the energy formula for a closed string becomes

$$
p_{0}=\sqrt{\left(\frac{n \tilde{R}}{\alpha^{\prime}}\right)^{2}+\left(\frac{w}{\tilde{R}}\right)^{2}+p_{\perp}^{2}+\frac{2}{\alpha^{\prime}}\left(N_{L}+N_{R}\right)}
$$

where $\tilde{R}=\alpha^{\prime} / R$ is the radius of the circle in the IIA description, whereas $n$ and $w$ are the original IIB Kaluza-Klein and winding numbers.

If we perform a boost with velocity $v$ along the $x^{1}$ direction, the energy in the boosted frame $\mathrm{F}^{\prime}$ (where the circle is no longer purely spatial) will read

$$
p_{0}^{\prime}=-\gamma v \frac{w}{\tilde{R}}+\gamma \sqrt{\left(\frac{n \tilde{R}}{\alpha^{\prime}}\right)^{2}+\left(\frac{w}{\tilde{R}}\right)^{2}+p_{\perp}^{2}+\frac{2}{\alpha^{\prime}}\left(N_{L}+N_{R}\right)},
$$

where $\gamma=1 / \sqrt{1-v^{2}}$. We now wish to compare this with the T-dual image of (25), which describes a theory with a $B$-field, compactified on a purely spatial circle of radius $R$. For this we apply the standard rules for T-duality in the presence of a $B$-field - see, e.g., [48]. Defining

$$
E_{a b}=g_{a b}+B_{a b}=\left(\begin{array}{cc}
-1 & R B_{01} \\
-R B_{01} & R^{2}
\end{array}\right)
$$

(in coordinates such that direction 1 has period $2 \pi$ ), and transforming according to

$$
E \rightarrow(a E+b)(c E+d)^{-1}
$$


with

$$
a=d=\left(\begin{array}{ll}
1 & 0 \\
0 & 0
\end{array}\right) \quad, \quad b=c=\left(\begin{array}{ll}
0 & 0 \\
0 & 1
\end{array}\right)
$$

leads to a new metric

$$
g_{a b}^{\prime \prime}=\left(\begin{array}{cc}
-1+B_{01}^{2} & -B_{01} / R \\
-B_{01} / R & 1 / R^{2}
\end{array}\right)
$$

and no $B$-field!. In the case of compact Euclidean time the result of such a Tduality is to map a straight torus with a $B$-field into a tilted torus without a $B$-field. The spectrum is of course invariant under this transformation, so it is still given by the mass formula (23), except that $w$ and $n$ are interpreted in the IIA theory as momentum and winding, respectively. One should note that the second term, linear in $B_{01}$, is no longer due to winding and the presence of a $B$-field, but to a mixing of momentum and energy resulting from the non-diagonal metric (30). From (23) we deduce that the energy is given by

$$
p_{0}^{\prime \prime}=-B_{01} \frac{w}{\tilde{R}}+\sqrt{\left(\frac{n \tilde{R}}{\alpha^{\prime}}\right)^{2}+\left(\frac{w}{\tilde{R}}\right)^{2}+p_{\perp}^{2}+\frac{2}{\alpha^{\prime}}\left(N_{L}+N_{R}\right)} .
$$

The final step is to compare this with (29). The frame $\mathrm{F}^{\prime \prime}$ in which the off-diagonal metric is written is such that one coordinate axis is along the compact direction, just as before the T-duality, even though this direction is no longer purely spatial. The other axis is along the original time direction. To compare with (29) we must change coordinates to those of the boosted coordinate system $\mathrm{F}^{\prime}$, bringing the metric back to a diagonal form. Applying the necessary transformation to the energy and momentum in frame $\mathrm{F}^{\prime \prime}$ one finds

$$
\left(\begin{array}{c}
p_{0}^{\prime} \\
p_{1}^{\prime}
\end{array}\right)=\frac{1}{\sqrt{1-B_{01}^{2}}}\left(\begin{array}{cc}
1 & 0 \\
-B_{01} & 1-B_{01}^{2}
\end{array}\right)\left(\begin{array}{c}
p_{0}-B_{01} p_{1} \\
p_{1}
\end{array}\right)=\left(\begin{array}{c}
\gamma p_{0}-\gamma v p_{1} \\
-v \gamma p_{0}+\gamma p_{1}
\end{array}\right)
$$

where in the second step we have identified $v=B_{01}$. This precisely adds the factor of $\gamma$ present in (29), and so completes the analysis. The identification of $B_{01}$ and $v$ is the natural analogue of the fact that under T-duality one has $E=2 \pi \alpha^{\prime} F_{01} \leftrightarrow v=\partial_{0} X^{1}$ for a D-brane. In particular, it leads us to conclude that $B_{01}$ has maximum value one- even in the absence of D-branes.

Returning to the NCOS setting, an immediate observation is that the description where $B$ (and not $E$ ) is made critical is the one that is more 'directly' T-dual to DLCQ IIA. Remember that this is the one description where one can easily work out the spectrum of closed strings, as in [12]. Open strings are of course only sensitive to the combination $E+B$.

\footnotetext{
${ }^{7}$ For related results see 19 .
} 


\subsection{The Decoupling}

We have just learned that the gauge-transformation which shifts $F_{01}$ into $B_{01}$ is Tdual to a boost. It is clear then that, when the $x^{1}$ direction is compact, this (large) gauge transformation, just like the boost, does not leave the spectrum invariant. On the other hand, it is evident that the physics must be the same in either gauge, since these are simply different descriptions of a single physical system. Now, as we have reviewed, in the $(E=0, B \neq 0)$ gauge the decoupling of closed strings with nonpositive winding number can be easily seen to follow from the energetics [12]. In the ( $E \neq 0, B=0$ ) gauge, on the other hand, there is no dependence of the closed string (free) spectrum on $E$, because closed strings are electrically neutral. We thus appear to have a contradiction: how could a decoupling take place in this gauge?

Let us examine the situation more closely. The standard mass-shell condition for a closed string (in the absence of $B$ ) implies that

$$
p_{0}=\sqrt{\left(\frac{w R}{\alpha^{\prime}}\right)^{2}+\left(\frac{n}{R}\right)^{2}+\frac{k_{\perp}^{2}}{h}+\frac{2}{\alpha^{\prime}}\left(N_{L}+N_{R}\right)} .
$$

We would like to extract from this expression a decoupling of all $w \leq 0$ states in the NCOS limit: $\epsilon \rightarrow 0$ with $\alpha^{\prime}=\epsilon \alpha_{e}^{\prime}, h=\epsilon\left(\alpha_{e}^{\prime}, k_{\perp}\right.$ fixed). It is obvious that the scaling of the electric field on the D1-brane, $E=1-\epsilon / 2$, is irrelevant. We see from (32) that in the $(E \neq 0, B=0)$ gauge we would expect all closed string states to decouple! In particular, at this level there does not seem to be any difference between states with positive and negative winding.

Inferring decoupling in this manner is clearly too naive. Eq. (32) certainly implies that, in the NCOS limit, the energy of closed strings with any winding number is much higher than that of open strings (the latter is of order $1 / \sqrt{\alpha_{e}^{\prime}}$ ). We should remember, however, that the open strings describe excitations of an $(N, 1)$ string, whose rest energy also should be taken into account. It is given by the standard BPS formula,

$$
2 \pi R T_{(N, 1)}=\frac{R}{\alpha^{\prime}} \sqrt{N^{2}+\frac{1}{g_{s}^{2}}},
$$

which clearly diverges in the NCOS limit,

$$
2 \pi R T_{(N, 1)} \simeq \frac{R|N|}{\alpha_{e}^{\prime} \epsilon} \rightarrow \infty .
$$

Starting with the $(N, 1)$ string (excited or not), there is thus plenty of energy available to create closed strings.

To make a fair comparison, then, we should allow for the appropriate subtraction also in the case of the closed strings. In this context it is important to remember that the sum of the number of electric flux units on the D1-brane and the closed string winding number, $W=N+w$, is conserved by interactions. When we compare energies of states with different values of $w$, we should consequently make sure that they belong to the same $W$ sector- otherwise the comparison is meaningless. Let us 
then fix a (positive) value of $W$ once and for all. From (32) and (33), it follows that (ignoring interactions) a state with the $(N, 1)$ string and a closed string with $w=0$ has total energy

$$
P_{0}=\epsilon^{-1} \frac{R|W|}{\alpha_{e}^{\prime}}+\epsilon^{-1 / 2} \sqrt{k_{\perp}^{2}+\frac{2}{\alpha_{e}^{\prime}}\left(N_{L}+N_{R}\right)}+\mathcal{O}(1),
$$

whereas if the closed string is wound, $w \neq 0$, the total energy of the state is

$$
P_{0}=\epsilon^{-1} \frac{R(|W-w|+|w|)}{\alpha_{e}^{\prime}}+\frac{|W-w| R}{2 \alpha_{e}^{\prime}}+\frac{\alpha_{e}^{\prime} k_{\perp}^{2}}{2|w| R}+\frac{N_{L}+N_{R}}{|w| R}+\mathcal{O}(\epsilon) .
$$

It should be noted that in the second term of (36) we have made use of the relation $g_{s}(W-w)=\sqrt{\epsilon}$, which follows from the quantization condition (2) for the nearcritical electric field on the brane, $E=1-\epsilon / 2$.

The first term in (35) is the 'rest energy' due to $W$, so it is evidently common to all states in the sector under consideration. Subtracting it, we see from (35) and (36) that the 'dynamical' energy diverges like $\epsilon^{-1 / 2}$ if $w=0$, diverges like $\epsilon^{-1}$ if $w<0$, and is finite only if $w>0$ (and $w<W$ ). The decoupling of all $w \leq 0$ states is now evident. In the $w>0$ case, the dynamical energy is given by

$$
P_{0}=\frac{(W-w) R}{2 \alpha_{e}^{\prime}}+\frac{\alpha_{e}^{\prime} k_{\perp}^{2}}{2 w R}+\frac{N_{L}+N_{R}}{w R},
$$

which agrees with the result (27) of Klebanov and Maldacena [12], except that $w$ has been replaced with $N=W-w$ in the numerator of the first term. This difference is partly due to the inclusion of the 'dynamical' energy of the $(N, 1)$ string.

We have thus succeeded in understanding the decoupling of closed strings with non-positive winding directly in the $(E \neq 0, B=0)$ gauge. Before closing this section, let us address another possible point of concern: given that the NCOS limit takes $g_{s} \rightarrow \infty$, do we really learn anything from (32) or (25), which describe the closed string spectrum of the free IIB theory? Notice that this is not an issue of continuing to strong coupling, since we are considering the full spectrum, not just BPS states. The essential point is that the effective closed string coupling constant for closed strings is not $g_{s}$, but $G_{o}^{2} \sqrt{\alpha_{e}^{\prime}}$, which remains finite in the limit. This has been shown in [5] by determining the cost of adding a handle to a worldsheet describing open string scattering to be $G_{o}^{4} \alpha_{e}^{\prime}$. In the next section we will understand this result from a different perspective.

\section{IIA/B Wound String Theory}

\subsection{Closed Strings}

In the previous section we have seen that the decoupling of closed strings with nonpositive winding number in the $1+1$ NCOS theory can be made explicit even in 
the absence of a $B$-field. The effect clearly does not depend on the presence of the D1-brane, either. So we learn that, as long as there is a compact dimension, one can meaningfully define an 'NCOS' limit of IIB (or IIA) string theory even in a vacuum with zero background fields and no D-branes. To be more precise, we consider IIA/B string theory compactified on a circle of radius $R$ along direction $x^{1}$, with metric (1), in the limit

$$
\delta \rightarrow 0, \quad g_{s}=\frac{G_{s}}{\sqrt{\delta}}, \quad l_{s}=L_{s} \sqrt{\delta}, \quad h=\delta, \quad \text { with } \quad G_{s}, L_{s}, R \quad \text { fixed }
$$

We have defined here a rescaled coupling constant and string length; their relation to the parameters $G_{o}^{2}, \alpha_{e}^{\prime}$ employed in [4, 5, 6] and in the previous sections will be discussed in Section 4.3. We now proceed to examine the theory obtained in the limit (38), which contains only closed strings.

To begin with, it is worth revisiting the decoupling argument from a slightly different perspective. Consider a scattering process in which the initial state has $C$ closed strings with winding numbers $w_{I}$ and momenta $p_{\mu I}=\left(p_{0 I}, n_{I} / R, p_{\perp I}\right)$, where $I=1, \ldots, C$ and the coordinates are such that the metric is given by (1). The corresponding quantities in the final state will be denoted by primed variables. As we have seen before, in the limit (38) with $p_{\perp}$ fixed, the energy of the individual strings diverges: it is

$$
p_{0}=\delta^{-1 / 2} \sqrt{p_{\perp}^{2}+\frac{2}{L_{s}^{2}}\left(N_{L}+N_{R}\right)}+\mathcal{O}(1)
$$

for an unwound closed string, and

$$
p_{0}=\delta^{-1} \frac{|w| R}{L_{s}^{2}}+\frac{L_{s}^{2} p_{\perp}^{2}}{2|w| R}+\frac{N_{L}+N_{R}}{|w| R}+\mathcal{O}(\delta)
$$

for a string with $w \neq 0$.

The important observation is that, for energy to be conserved in the scattering process, it must do so separately at each order in an expansion in powers of $\delta$. Equating the coefficient of $\delta^{-1}$ in the initial and final energies we obtain the requirement

$$
\left|w_{1}\right|+\ldots+\left|w_{C}\right|=\left|w_{1}^{\prime}\right|+\ldots+\left|w_{C^{\prime}}^{\prime}\right| .
$$

On the other hand, the statement of winding number conservation reads

$$
w_{1}+\ldots+w_{C}=w_{1}^{\prime}+\ldots+w_{C^{\prime}}^{\prime}
$$

Now, suppose all of the initial strings have positive winding, $w_{I}>0$. Then the only way that (41) and (42) can be simultaneously satisfied is if all of the final strings also have non-negative winding, $w_{I}^{\prime} \geq 0$. Moreover, (39) shows that if $w_{I}^{\prime}=0$ for some $I$ then there would be a contribution of $\mathcal{O}\left(\delta^{-1 / 2}\right)$ to the final energy, which would have no counterpart in the initial energy. We therefore conclude that no strings with non-positive winding can be produced in the scattering event. 
Notice that the restriction on the initial state was put in by hand. Clearly, if some of the incoming strings have negative (or zero) winding, then these can interact with positively-wound strings. The important point is that one can consistently restrict attention to the subsector where all closed strings have strictly positive winding§, and within it, conservation of the leading, $\mathcal{O}\left(\delta^{-1}\right)$ piece of the energy is guaranteed by winding-number conservation. It is thus sensible to define a 'dynamical' energy by removing this divergent contribution: $\hat{p}_{0}=p_{0}-w R / \delta \alpha^{\prime}$. The total dynamical energy is conserved and finite.

Given the fact that they contain only (positively) wound strings, it is natural to call these theories the Type II Wound String Theories (or Wound IIA/B, for short). We will consequently refer to the limit (38) as the Wound limit.

It should be noted that the decoupling argument we have given above mirrors the standard discussion in the infinite-momentum frame (IMF) [49, 50]. This is no accident: as we have seen in Section 2, (ordinary) $\mathrm{T}_{1}$-duality maps the Wound IIA/B theory onto IIB/A in the IMF (with a compact longitudinal direction?), with the Wound winding $W$ determining the IMF longitudinal momentum, $P_{1}=W / \tilde{R} \rightarrow \infty$. This is obviously conserved, so it is natural again to subtract its (divergent) contribution to the energy. The remaining 'dynamical' energy is precisely what corresponds to the finite lightcone energy $P_{+}^{\prime}$ in the the frame $\mathrm{F}^{\prime}$ where the circle is null (and $P_{-}^{\prime}=W / \tilde{R}_{-}$is finite). We also understand by now that the role of the near-critical $B_{01}$-field employed in [12] is merely to effect this subtraction. The restriction to the lowest energy subsector of the IMF theory is standard; it is necessary to match the degrees of freedom of a direct DLCQ description (where all momenta are strictly positive from the beginning) [51, 52].

The dynamical energy of a Wound string is

$$
\hat{p}_{0}=\frac{L_{s}^{2} p_{\perp}^{2}}{2 w R}+\frac{N_{L}+N_{R}}{w R} .
$$

Notice that, compared to the Klebanov-Maldacena result, Eq. (27), the above expression is missing the first term. That term represents a rescaled winding energy, and it can be seen to play the role of a Newtonian mass in the non-relativistic-type expression (43). But again, since the total winding number (just like the total Newtonian mass) is conserved, inclusion of this term, albeit natural, does not affect the dynamics: it constitutes an irrelevant shift of the energy. The naive string tension inferred from this Newtonian mass is

$$
\hat{T}_{F}=\frac{1}{2 \pi L_{s}^{2}}
$$

\footnotetext{
${ }^{8}$ To be precise, we should note that unwound closed strings can be considered as long as they have $N_{L}=N_{R}=p_{\perp}=0$. Their energy is then finite in the limit (38),$p_{0}=|n| / R$. Such strings would clearly not make any non-trivial contribution to the dynamics, so they can be ignored.

${ }^{9}$ We are employing the term IMF here to refer to the frame $\mathrm{F}$ where the circle is purely spatial: the momentum is infinite in this frame as a result of the shrinking radius. This frame is related to the DLCQ frame $\mathrm{F}^{\prime}$ by a large boost.
} 
This agrees with the usual result, and consequently differs from the tension discussed in 12 by a factor of two. In other words, (27) is a little peculiar: the first term is only half of the Newtonian mass inferred from the second term. Even so, it was shown in [12] that (27) is in exact agreement with the corresponding $1+1 \mathrm{SYM}$ result. A further peculiarity of (43) is the presence of the second term, which amounts to a contribution to the rest mass of the string distinct from the Newtonian mass discussed above.

To summarize, we have shown that upon compactification on a (purely spatial) circle, there exists a meaningful 'NCOS' limit of IIA/B string theory in the absence of D-branes. This yields a theory of closed strings with strictly positive winding, Wound IIA/B, which is T-dual to DLCQ IIB/A. Agreement between the corresponding scattering amplitudes is guaranteed by the usual rules of T-duality - we will elaborate on this point in the next subsection.

\subsection{Closed String Amplitudes}

Since the closed string sector of Wound IIA/B is T-dual to that of DLCQ IIB/A, we expect the scattering amplitudes of these closed strings to be well-defined in the Wound limit (38). The energy formula (43) plus conservation of winding number shows that the system can be described as non-relativistic particles with masses $w_{I} R$ and an internal energy with the spectrum of a harmonic oscillator. In addition, there is an extra conserved charge $n$, the momentum along the circle, which appears only through the constraint (24). These particles move in eight transverse dimensions and their scattering is given by the Wound limit of the usual scattering amplitudes for strings wound around a circle (see, e.g., 41).

When string theory is compactified on a circle one has to distinguish between leftand right-moving momenta along the compact direction, defined as

$$
k_{L, R}=\frac{n}{R} \pm \frac{w R}{\alpha^{\prime}}
$$

A generic amplitude depends on the Lorentz invariant products $\alpha^{\prime} k_{i L, R} k_{j L, R}$, and the important observation is that these are finite in the limit. For example, in the case of external particles with zero momentum along the circle one has $k_{i L} k_{j L}=k_{i R} k_{j R}$, which in the limit reduces to

$$
\begin{aligned}
\alpha^{\prime} k_{i L} k_{j L}= & \frac{2 w_{i} w_{j} R^{2}}{\alpha^{\prime}}+\frac{w_{i}}{w_{j}}\left(L_{s}^{2}\left(k_{j}^{\perp}\right)^{2}+\mu_{j}^{2}\right)+\frac{w_{j}}{w_{i}}\left(L_{s}^{2}\left(k_{i}^{\perp}\right)^{2}+\mu_{i}^{2}\right) \\
& -2 L_{s}^{2} k_{1}^{\perp} k_{j}^{\perp}-\frac{2 w_{i} w_{j} R^{2}}{\alpha^{\prime}} \\
= & \mu_{j}^{2} \frac{w_{i}}{w_{j}}+\mu_{i}^{2} \frac{w_{j}}{w_{i}}+L_{s}^{2} w_{i} w_{j}\left(\frac{1}{w_{j}} k_{j}^{\perp}-\frac{1}{w_{i}} k_{i}^{\perp}\right)^{2},
\end{aligned}
$$

\footnotetext{
${ }^{10}$ These particles can be thought of as bound states of $w_{I}$ particles with winding number one.
} 
where $\mu_{j}^{2}=2\left(N_{L}+N_{R}\right)$, and we have taken into account the fact that the transverse metric is $g_{i j}=\left(L_{s} / l_{s}\right)^{2} \delta_{i j}$. Note that the dangerous terms that behave as $1 / \alpha^{\prime}$ cancel. This means that one can safely take the Wound limit (38). For example, in the case of a four-point amplitude of massless particles, the Mandelstam variables are

$$
\begin{aligned}
& \alpha^{\prime} s=-\left(k_{1, L}+k_{2, L}\right)^{2}=L_{s}^{2} w_{1} w_{2}\left(\frac{1}{w_{2}} k_{2}^{\perp}-\frac{1}{w_{1}} k_{1}^{\perp}\right)^{2}=L_{s}^{2} w_{1} w_{2}\left(v_{1}^{\perp}-v_{2}^{\perp}\right)^{2} \\
& \alpha^{\prime} t=-\left(k_{1, L}-k_{3, L}\right)^{2}=L_{s}^{2} w_{1} w_{3}\left(\frac{1}{w_{3}} k_{3}^{\perp}-\frac{1}{w_{1}} k_{1}^{\perp}\right)^{2}=L_{s}^{2} w_{1} w_{2}\left(v_{1}^{\perp}-v_{3}^{\perp}\right)^{2} \\
& \alpha^{\prime} u=-\left(k_{1, L}-k_{4, L}\right)^{2}=L_{s}^{2} w_{1} w_{4}\left(\frac{1}{w_{4}} k_{2}^{\perp}-\frac{1}{w_{1}} k_{1}^{\perp}\right)^{2}=L_{s}^{2} w_{1} w_{2}\left(v_{1}^{\perp}-v_{4}^{\perp}\right)^{2}
\end{aligned}
$$

where $v_{i}^{\perp}=k_{i}^{\perp} / w_{i}$ is the transverse velocity of the string. In the Wound limit the form of the amplitude is left intact; the only modification is to replace $s, t, u$ by the formulas (47). For instance, one can take two strings with winding number $w_{1}$, $w_{2}$, a relative velocity $v_{12}$ in direction $\hat{8}$, and relative momentum $p_{12}$ in the other 7 dimensions. Expanding for small $\left|p_{12}\right|$ and Fourier transforming to position space one would obtain the dimensional reduction of the usual $v_{12}^{4} / r^{7}$ potential for two D0branes, in a manner similar to [53]. Here we do not need to take $v_{12}$ small, since that is included as part of the Wound limit.

Let us now examine what happens with one-loop corrections. As shown in [45], in the T-dual language the torus amplitude has a finite limit. In our example of four massless particles, this one-loop amplitude is given by [45, 43]

$$
\begin{aligned}
A^{(4)}= & K \int \frac{d^{2} \tau}{(\operatorname{Im} \tau)^{2}} \int \prod_{a=1}^{3} \frac{d^{2} \nu_{a}}{\operatorname{Im} \tau} \mathcal{I} \\
\mathcal{I}= & \prod_{a, b} E\left(\nu_{a b}, \tau\right)^{\alpha^{\prime} k_{a R} \cdot k_{b R} / 2} \bar{E}\left(\nu_{a b}, \tau\right)^{\alpha^{\prime} k_{a L} \cdot k_{b L} / 2} e^{\frac{\pi \alpha^{\prime}}{\operatorname{Im} \tau}\left(\sum_{a} k_{a}^{\perp} \operatorname{Im} \nu_{a}\right)} \mathcal{S} \\
\mathcal{S}= & g_{s}^{2} \sqrt{\operatorname{Im} \tau} \frac{\sqrt{\alpha^{\prime}}}{R} \sum_{n, m} e^{-\pi \operatorname{Im} \tau\left(\frac{m^{2} R^{2}}{\alpha^{\prime}}+\frac{\alpha^{\prime} n^{2}}{R^{2}}\right)-2 \pi i m n \operatorname{Re} \tau} \times \\
& \times e^{\left.-i \pi\left(\frac{\alpha^{\prime}}{R} n\left(k_{L a}^{1} \bar{\nu}_{a}-k_{R a}^{1} \nu_{a}\right)+m R\left(k_{L a}^{1} \bar{\nu}_{a}+k_{R a}^{1} \nu_{a}\right)\right)\right)}
\end{aligned}
$$

where $E\left(\nu_{a b}, \tau\right)=\Theta_{1}(\nu \mid \tau) / \Theta_{1}^{\prime}(0 \mid \tau)$ and $\nu_{a b}=\nu_{a}-\nu_{b}$. Also we included in $K$ the kinematic factor and all the constants which are not important for our purpose.

The function $\mathcal{S}$ is manifestly T-duality invariant, including the coefficient $g_{s}^{2} \sqrt{\alpha^{\prime}} / R$ in front. In order to take $\alpha^{\prime} \rightarrow 0$ it is convenient to perform a Poisson resummation on $n$, which yields 45

$$
\mathcal{S}=g_{s}^{2} \sum_{m, n} e^{-\frac{\pi R^{2}}{\alpha^{\prime} \mathrm{m} \tau}\left\{\left(n+m \tau-\frac{\alpha^{\prime}}{R} k_{R a}^{1} \nu_{a}\right)\left(n+m \bar{\tau}+\frac{\alpha^{\prime}}{R} k_{L a}^{1} \bar{\nu}_{a}\right)+\frac{\alpha^{\prime 2}}{4 R^{2}}\left(k_{L a}^{1} \bar{\nu}_{a}+k_{R a}^{1} \nu_{a}\right)^{2}\right\}} .
$$

Using $k_{L, R a}^{1}= \pm m_{a} R / \alpha^{\prime}$ we obtain

$$
\mathcal{S}=g_{s}^{2} \sum_{m, n} e^{-\frac{\pi R^{2}}{\alpha^{\prime} \mathrm{Im} \tau}\left|n+m \tau-m_{a} \nu_{a}\right|^{2}}
$$


As argued by Bilal 45, in the $\alpha^{\prime} \rightarrow 0$ limit this becomes a delta-function,

$$
\mathcal{S}=g_{s}^{2} \alpha^{\prime} \frac{\operatorname{Im} \tau}{R^{2}} \sum_{m, n} \delta^{(2)}\left(n+m \tau-m_{a} \nu_{a}\right)
$$

which converts one of the $\nu_{a}$ integrals into a sum. Then, when $\alpha^{\prime} \rightarrow 0$ the amplitude vanishes unless we scale $g_{s} \sim G_{s} / l_{s} \rightarrow \infty$, as in the Wound limit (38). We have thus shown that the argument in [5] stating that the handles are weighted by $G_{s}^{2}$ and not by $g_{s}^{2}$ has a T-dual counterpart in the argument of 45.

\subsection{D-branes and Couplings}

Having understood the effect of the Wound limit (38) on closed strings from various different perspectives, let us now re-examine its effect on D-branes. As discussed in Section 3.3, the energy of a D1-brane wrapped on the Wound circle which carries $N$ units of electric flux is given by (33). For $N \neq 0$ this becomes

$$
2 \pi R T_{(N, 1)}=\frac{R|N|}{L_{s}^{2} \delta}+\frac{R}{2|N| G_{s}^{2} L_{s}^{2}}+\mathcal{O}(\delta)
$$

in the Wound limit. The first term is of the same order as the divergent term in (40), reflecting the fact that electric flux on the brane is equivalent to fundamental string winding. In the remainder of the paper, we will find it most convenient to use the latter terminology. Again, the key point is that as long as $N>0$, one can subtract the divergent term on account of conservation of the (total) winding number. We thus learn that D-strings exist in the Wound theory only if they have strictly positive (F-string) winding. This again is easy to understand in the T-dual language: only D0-branes with strictly positive longitudinal momentum are part of the DLCQ IIA theory.

It is by now evident that the various setups which have hitherto been known as ' $(p+1)$-dimensional NCOS theories' [4, 5, 6] are simply different truncations of the state space of the corresponding Wound theory to the subspace of states containing a $\mathrm{D} p$-brane extended along the Wound direction. We also understand that the need to turn on an electric field in the $\mathrm{D} p$-brane worldvolume (which then becomes critical in the limit) is merely an expression of the fact that only objects carrying strictly positive F-string winding remain in the spectrum $\square$.

We are now ready to address the question of how the Wound closed string parameters $G_{s}, L_{s}$ introduced in (38) are related to the NCOS open string quantities employed in [4, 5, [6] and Sections 2 and 3 of the present paper. In the standard NCOS discussion one infers from the formulas of [3, 17] and the electric-flux quantization condition (2) that open strings describing the dynamics of a single D1-brane carrying $N$ units of F-string winding interact with strength $G_{o}^{2}=1 / N$, and their

\footnotetext{
${ }^{11}$ We should note that this requirement also applies to NS5-branes, which will be present in the spectrum of the theory only if they carry positive fundamental string winding.
} 
tension is set by a parameter $\alpha_{e}^{\prime}$. With this as a starting point it might be tempting to define a closed string coupling using the standard formula $G_{s}=G_{o}^{2}$. However, in our context this would clearly not be an appropriate definition, because $G_{o}^{2}$ is a function of the parameters $N$ and $p$ that characterize a specific brane configuration of the theory, whereas the closed string coupling $G_{s}$ should be universal. Related to this, the rescaling of the transverse metric (1) in the standard NCOS limit (4) is in

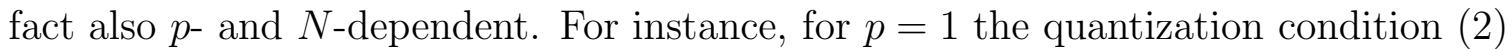
implies that $\epsilon=1 /\left(N g_{s}\right)^{2}$, as seen in (5). Again, whereas this is not unreasonable for the purpose of [4, [5, [6], which is to consider one brane configuration at a time, it is not convenient now that we have come to realize that these are all just different states in the same underlying theory.

The essential content of the limits (44) and (38) is the scaling $g_{s} \rightarrow \infty, l_{s} \rightarrow 0$, holding $g_{s} l_{s}$ fixed. From this observation it follows that

$$
G_{s} L_{s}=g_{s} l_{s}=G_{o}^{2} \sqrt{\alpha_{e}^{\prime}} .
$$

It is important to understand that in the right-hand side the dependence of the parameters $G_{o}^{2}$ and $\sqrt{\alpha_{e}^{\prime}}$ on the specific brane quantities $N$ and $p$ cancels out. Similarly, taking into account the different rescalings of the metric we infer that the perpendicular momenta in the two descriptions are related through

$$
L_{s}^{2} p_{\perp}^{2}=\alpha_{e}^{\prime} k_{\perp}^{2} .
$$

We have in fact already made implicit use of this relation when comparing (43) with (27).

In a certain sense, the parameter that truly characterizes the closed string sector is the dimensionful quantity (53), which is held fixed in the Wound limit. For a perturbative expansion this dimensionful coupling should be compared with some other relevant scale in order to form a dimensionless expansion parameter. Since we always have the compactification radius $R$ at our disposal, it makes physical sense to focus on $g_{s} l_{s} / R$. That this is the combination which controls the closed string loop expansion from a nine-dimensional perspective is made evident by the result (51) for the torus amplitude, which shows the addition of a handle costs a factor of $\left(g_{s} l_{s} / R\right)^{2}$. This is not surprising, for $g_{s} l_{s} / R$ is in fact the coupling constant of the dual DLCQ theory. It would perhaps seem natural then to give this quantity the name $G_{s}$, which in view of (53) amounts to fixing $L_{s} \equiv R$. We choose not to do this, however, because it might be a source of confusion, given that there are processes in the theory where this is not the relevant quantity. Examples include adding a handle to a worldsheet describing open string scattering, as in [5], or high-energy $(E \gg 1 / R)$ closed string scattering, where the relevant expansion parameter is in effect $g_{s} l_{s} E$ (meaning that the theory is strongly-coupled when $g_{s} l_{s} E \gg 1$ ). The fact that the Wound theory lacks a definite dimensionless coupling is presumably related to the fact that the Wound limit removes the dilaton from the spectrum of the theory.

Let us now return to the discussion of the D-branes present in the Wound spectrum. It follows from (52) that the 'dynamical' tension of a Wound D1-brane with 
$N$ units of winding is

$$
\hat{T}_{D 1, N}=\frac{1}{4 \pi N G_{s}^{2} L_{s}^{2}} .
$$

Using (53) and (6) we can rewrite this as

$$
\hat{T}_{D 1, N}=\frac{1}{4 \pi G_{o}^{2} \alpha_{e}^{\prime}}
$$

which agrees with the standard formula except for a factor of two discussed already in [12]. The form (55), on the other hand, has the advantage of manifestly exhibiting the $N$-dependence of the tension. Notice that the heaviest D-string is the one with a single unit of F-string winding. The dependence of (55) on $N, G_{s}$ and $L_{s}$ might seem peculiar, but it is precisely as needed for the energy of the D-string to agree with that of its T-dual image in DLCQ IIA. Indeed, using the T-duality formulas obtained in Section 2, one finds that

$$
2 \pi R \hat{T}_{D 1, N}=\frac{\tilde{R}_{-}}{2 N}\left(\frac{1}{\tilde{g}_{s} \tilde{l}_{s}}\right)^{2}=P_{+}^{\prime},
$$

which is the lightcone energy of a D0-brane carrying $N$ units of longitudinal momentum.

The preceding discussion can clearly be generalized to $\mathrm{D} p$-branes with $p>1$ which wrap the Wound circle. The simplest way to do this is to start with the D1-brane carrying $N>0$ units of (F-string) winding in Wound IIB with coupling constant $\tilde{G}_{s}$, and compactify directions $x^{2}, \ldots, x^{p}$ on a rectangular torus with radii $\tilde{r}_{2}, \ldots, \tilde{r}_{p}$. Ordinary T-duality along these transverse directions [6, 34] then converts this into a $\mathrm{D} p$-brane wrapped on a torus of radii $R$ and $r_{i}=L_{s}^{2} / \tilde{r}_{i}, i=2, \ldots, p$. Equating the energy of the initial and final configurations yields the condition

$$
\frac{2 \pi R N}{4 \pi N G_{s}^{2} L_{s}^{2}}=(2 \pi)^{p} R r_{2} \cdots r_{p} \hat{T}_{D p}
$$

where $\hat{T}_{D p}$ denotes the tension of the $p$-brane after subtraction of the divergent $\mathcal{O}\left(\delta^{-1}\right)$ part. Using the fact that the coupling constant of the theory after T-duality is [6, 34]

$$
G_{s}=\tilde{G}_{s} \frac{r_{2} \cdots r_{p}}{L_{s}^{p-1}}
$$

the tension of the brane follows as

$$
\hat{T}_{D p, \nu}=\frac{1}{2(2 \pi)^{p} \nu G_{s}^{2} L_{s}^{p+1}},
$$

where in the last step we have defined a dimensionless parameter

$$
\nu \equiv \frac{N L_{s}^{p-1}}{r_{2} \cdots r_{p}},
$$

\footnotetext{
${ }^{12}$ These are held fixed in the Wound limit, so in the closed string metric relevant for the underlying IIB description the corresponding proper radii shrink to zero size (they are fixed in units of $l_{s}$ ).
} 
which expresses the (strictly positive) density of F-string winding carried by the $p$ brane. This is clearly the quantity which should be held fixed if we wish to take the decompactification limit $r_{i} \rightarrow \infty$. Again, the tension (60) can be written in the more familiar but less transparent form

$$
\hat{T}_{D p, \nu}=\frac{1}{2(2 \pi)^{p} G_{o, p}^{2} \alpha_{e}^{\prime(p+1) / 2}},
$$

which uses the effective coupling $G_{o, p}^{2}$ that governs interactions of open strings on the $p$-brane, and is related to $G_{o}^{2} \equiv G_{o, 1}^{2}$ through a formula analogous to (59).

It follows from the preceding discussion that a Wound $\mathrm{D} p$-brane extended along $x^{1}$, with $N$ units of longitudinal (F-string) winding, is $\mathrm{T}_{1}$-dual to a DLCQ IIA/B $\mathrm{D}(p-1)$-brane with $N$ units of longitudinal momentum. One way to see this is to apply the $\mathrm{T}_{2 \ldots}$-duality argument to the known $p=1$ case. Notice that this procedure yields only branes which are not wrapped on the null circle. The DLCQ IIA/B theories also include longitudinally wrapped $\mathrm{D} p$-branes, and these are clearly $\mathrm{T}_{1}$-dual to $\mathrm{D}(p-1)$-branes in the Wound theory which are transverse to the Wound circle. The simplest case is that of the D0-brane in IIA Wound: its rest energy in the parent description is $1 / g_{s} l_{s}$, which is finite and equal to $1 / G_{s} L_{s}$ in the Wound limit. By transverse T-duality, one obtains all other transverse $\mathrm{D} p$-branes, and their tensions are found to be

$$
T_{D p}^{\perp}=\frac{1}{(2 \pi)^{p} G_{s} L_{s}^{p+1}}
$$

which unlike (60) agrees with the naive expectation.

Excitations of such branes will of course be described by open strings which end on them. It is interesting to note, however, that due to the familiar decoupling argument these open strings must necessarily have positive winding number along $x^{1}$. So these are not standard D-branes - in particular, the usual massless modes associated with quantum fluctuations of their positions are missing! As a matter of fact, the ground state configuration for these branes includes at least one F-string attached to them, since their total winding number $w$ is as always $T_{1}$-dual to the (necessarily positive) longitudinal momentum of the longitudinally wrapped DLCQ brane, $p_{-}^{\prime}=w / R$. In the parent type II string theory, the total energy of the combined brane-string system is thus divergent, but becomes finite after the usual subtraction.

Notice that one can also consider states with more than one type of brane present. For instance, a DLCQ IIA state with a D0- and a (transverse) D4-brane is mapped onto a IIB Wound state which includes both a D1-brane and a D5-brane (with the electric field on both branes becoming critical simultaneously). We emphasize that these two objects do not give rise to two decoupled theories: they can interact through the exchange of wound closed strings. We leave a study of these interactions to future work. 


\section{Wound IIA/B Theory on a Transverse Torus}

Since the Wound IIA/B theory is $\mathrm{T}_{1}$-dual to DLCQ IIB/A, it is a part of the wellknown duality web of DLCQ IIA/M-theory [27, 28, 29], 30]. In this section we examine this web of dual descriptions for Wound/DLCQ IIA/B theory compactified on a transverse $\mathbf{T}^{p-1}$, for $1 \leq p \leq 5$. The parameters of the Wound and DLCQ theories are in all cases related through the T-duality formulas obtained in Section 2:

$$
\tilde{R}_{-}=\frac{L_{s}^{2}}{R_{1}}, \quad \tilde{g}_{s}=G_{s} \frac{L_{s}}{R_{1}}, \quad \tilde{l}_{s}=L_{s}
$$

It is also of interest to examine the effect of $\mathrm{S}$ - and transverse T-duality, to incorporate in the discussion the known S-duals of the various NCOS theories [6, 34], and the Seiberg argument [29, 30] for DLCQ M-theory on a transverse $\mathbf{T}^{p}$. The knowledge gained in Section 19 will allow us to make some new inferences about these other descriptions. The situation for $p=1,3,5$ is summarized in Fig. 3, while Fig. 4 displays the cases $p=2,4$. Of course, the theories encountered at different values of $p$ are all connected through transverse T-dualities followed by decompactification of some directions. As we will also emphasize, the various NCOS theories simply correspond to different states in the universal Wound IIA/B theory.

We would like to single out a few of the theories in this duality web, and write down the explicit dictionary between their parameters and those of the Wound theory. Which theories are interesting depends on the value of $p$, so we will discuss each case separately. The case $p=1$ has already been discussed at length in Section 2, so we will omit it here. For the other cases, we will only discuss theories which are 'good' dual descriptions of the $\epsilon \rightarrow 0$ physics, in the sense that the relevant parameters are finite in this limit. The dual DLCQ IIA, DLCQ IIB and DLCQ M theories are good descriptions for all values of $p$, but we will not write down their parameters explicitly since the relevant formulas are essentially identical to those given in Section 2. In the discussion to follow we will denote the coordinate radii of the transverse cycles of the torus by $r_{i}, i=2, \ldots, p-1$. These are held fixed in the wound limit (recall that the effective Wound metric is simply $G_{\mu \nu}=\eta_{\mu \nu}$ ), which means that the corresponding proper radii $R_{i}=r_{i} \sqrt{\epsilon}$ in the parent type II description shrink to zero size in the limit (they are, however, fixed in units of $l_{s}$ ).

\section{$5.1 p=3$}

In the D3-brane case, the parameters of the theory termed $\overline{\text { IIB }}$ in Fig. 3 are related to those of the S-dual Wound theory through

$$
\bar{g}_{s}=G_{s}^{-1} \sqrt{\epsilon} \rightarrow 0, \quad \bar{l}_{s}=\sqrt{G_{s}} L_{s} \epsilon^{1 / 4} \rightarrow 0, \quad \bar{R}_{1}=R_{1}, \quad \bar{R}_{2,3}=r_{2,3} \sqrt{\epsilon} \rightarrow 0 .
$$

As seen in the figure, a collection of $K$ Wound D3-branes with $N$ units of F-string winding are mapped onto $K \overline{\mathrm{IIB}} \mathrm{D} 3$-branes with $N$ units of $\mathrm{D}$-string winding. The theory on the three-branes is thus a $U(K)(3+1)$-dimensional gauge theory with $N$ 


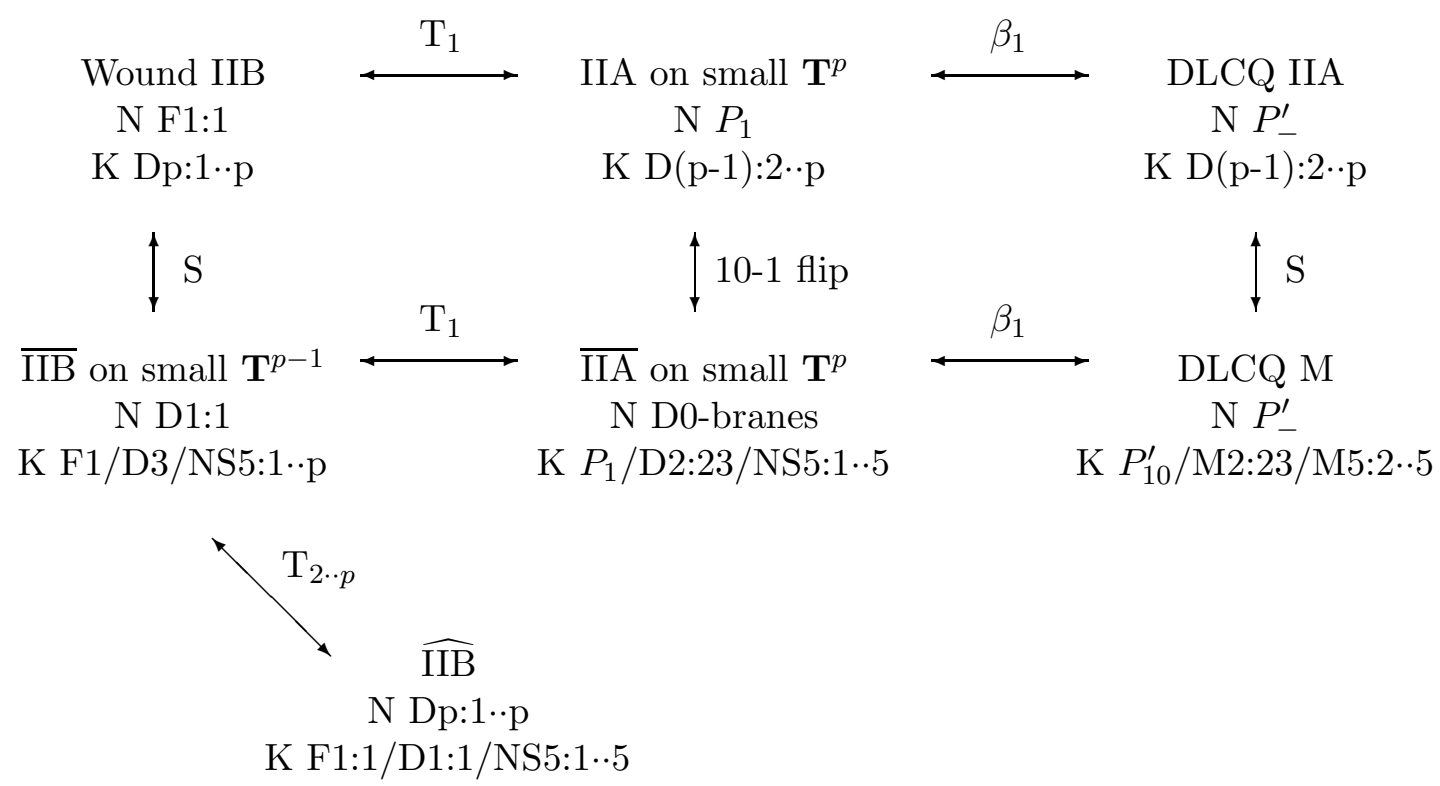

Figure 3: A portion of the duality web for Type II Wound/DLCQ theories on a transverse $\mathbf{T}^{p-1}$ for $p=1,3,5$, including the images of $K$ Wound $\mathrm{D} p$-branes and $N$ units of F-string winding in the various descriptions. As explained in Section $4, N$ must be strictly positive, but $K$ is arbitrary. The Wound IIB setup in the upper-left corner of the diagram has hitherto been known as $(p+1)$-dimensional NCOS theory. Non-vanishing-size compactifications are not mentioned. $\beta_{1}$ denotes a boost along $x^{1}$ together with a change of units. $P_{i}$ stands for Kaluza-Klein units of momentum along $x^{i}$. The notation Xq: $i_{1} \cdot i_{q-1}$ indicates an Xp-brane wrapping the $i_{1}, \ldots, i_{q-1}$ cycles of the torus. Triple $K$-entries apply respectively to the cases $p=1,3,5$. Seiberg's derivation of a non-perturbative (Matrix) formulation for DLCQ IIA on a transverse $\mathbf{T}^{p-1}$ (DLCQ $\mathrm{M}$ on a transverse $\mathbf{T}^{p}$ ) follows the horizontal arrows in the top (middle) line of the diagram, and then proceeds down and diagonally to arrive at the $\widehat{\mathrm{IIB}}$ theory. See text for further discussion.

units of $F_{23}$-flux. As explained in [5], the scaling of $\bar{g}_{s}$ and $\bar{l}_{s}$ seen in (65) is precisely the Seiberg-Witten scaling [3] which yields non-commutative super-Yang-Mills theory (NCYM). Just like in the Wound description, the proper radii of the non-commutative $x^{2}, x^{3}$ directions are fixed when measured in the open string metric relevant to the NCYM theory.

As seen in (65), the proper radii $R_{2,3}$ (measured in the underlying $\overline{\text { IIB }}$ metric) shrink to zero size in the Wound/DLCQ limit, so it is natural to take the final step in the Seiberg argument [29, 30], following the diagonal arrow in Fig. 3 ( $\mathrm{T}_{23}$-duality) to arrive at a $\widehat{\mathrm{IIB}}$ theory defined on a finite-size three-torus, with parameters

$$
\hat{g}_{s}=\frac{L_{s}^{2}}{r_{2} r_{3}}, \quad \hat{l_{s}}=\sqrt{G_{s}} L_{s} \epsilon^{1 / 4} \rightarrow 0, \quad \hat{R}_{1}=R_{1}, \quad \hat{R}_{2,3}=\frac{G_{s} L_{s}^{2}}{r_{2,3}} .
$$

The Wound IIB D3- and F1-branes now become $K$ D1-branes and $N$ D3-branes, so we are dealing with $(3+1)$-dimensional $U(N)$ (ordinary) SYM with $K$ units of 
$F_{23}$-flux. The Yang-Mills coupling for the theory is

$$
\hat{g}_{Y M}^{2}=2 \pi \hat{g}_{s}=\frac{2 \pi L_{s}^{2}}{r_{2} r_{3}}
$$

Clearly, there is nothing that prevents us from setting $K=0$, neither in the case of $\overline{\mathrm{IIB}}$, nor in the case of $\widehat{\mathrm{IIB}}$. In the former case we notice that D1-branes with strictly positive (D-string) winding along $x^{1}$ can be emitted at a finite energy cost into the bulk, where they can be studied on their own. It is thus sensible to consider this theory even in the absence of D3-branes (i.e., $K=0$ ): it is just the S-dual of the Wound IIB theory without any D-branes. We will refer to this as the Wound D-string theory. Notice that these two theories are really distinct: the strong/weak-coupling self-duality of the parent IIB theory is not inherited by the Wound IIB theory, because the wound limit scales $g_{s} \rightarrow \infty$.

Turning to the case of $\widehat{\mathrm{IIB}}$, where the $K$ D3-branes have turned into $K$ D-strings and the $N$ D1-strings have turned into $N$ D3-branes, the possibility for the wound D3-branes to emit a wound string into the bulk becomes a rather mundane symmetry breaking process. This is well-understood in the Matrix context, and is the obvious analog of the $1+1$ SYM breaking studied in [12 and reviewed in Section 2. In this description it is therefore also evident that there is nothing peculiar about the case $K=0$ (where all the wound strings have been emitted). In fact, this is nothing but the Matrix model conjectured to provide a non-perturbative description of DLCQ M-theory on a transverse $\mathbf{T}^{3}$ [27, 54].

One curious aspect of (66) and (67) is the fact that the coupling constant in this description is independent of the Wound coupling. Notice, however, that this does not lead one to the contradiction of having two manifestly distinct weakly-coupled descriptions of the same underlying physical system: simultaneously requiring that $G_{s} \ll 1$ and $\hat{g}_{Y M}^{2} \ll 1$ can be seen from (66) to imply that $r_{2,3} \ll L_{s}$, which means the Wound theory is not really amenable to a direct perturbative analysis.

\section{$5.2 p=5$}

In the case of D5-branes, the $\overline{\mathrm{IIB}}$ theory parameters are again given by (65), except that there are two more transverse directions, $x^{4}, x^{5}$. As noted in Fig. 3, the $K$ Wound D5-branes and $N$ F-strings are now mapped onto $K \overline{\mathrm{IIB}}$ NS5-branes and $N$ units of D-string winding. The latter manifest themselves as $N$ units of $\mathcal{F}_{01}$-flux in the NS5 worldvolume, where $\mathcal{F}$ is the two-form field strength (which is S-dual to $F$ on the original D5-branes). As explained in [6, 33], $\mathcal{F}$ (or equivalently, the Ramond-Ramond $C_{01}$ potential) becomes critical in the Wound limit, which together with the scaling (65) yields the so-called OD1 theory, whose excitations are open D-strings ending on the NS5-branes. Interactions of these open D-strings are governed by the coupling [6]

$$
G_{D 1}=\frac{\hat{g_{s}}}{\sqrt{\epsilon}}=\frac{1}{G_{s}},
$$


and the theory has a length parameter

$$
L_{D 1}=\frac{\hat{l_{s}}}{\epsilon^{1 / 4}}=\sqrt{G_{s}} L_{s} .
$$

Again, it is natural to take the next step in the Seiberg argument [29, 30], carrying out a $\mathrm{T}_{2345}$-duality transformation to arrive at a $\widehat{\mathrm{IIB}}$ theory defined on a finite-size five-torus, with parameters

$$
\hat{g_{s}}=\frac{1}{G_{s}} \frac{L_{s}^{4}}{r_{2} r_{3} r_{4} r_{5}} \epsilon^{-1 / 2} \rightarrow \infty, \quad \hat{l_{s}}=\sqrt{G_{s}} L_{s} \epsilon^{1 / 4} \rightarrow 0, \quad \hat{R}_{1}=R_{1}, \quad \hat{R}_{i}=\frac{G_{s} L_{s}^{2}}{r_{i}},
$$

in the presence of $K$ NS5-branes and $N$ D5-branes. This is the OD5 theory of [6, 33], whose excitations are described by open D5-branes ending on the NS5 worldvolume interacting with strength

$$
G_{D 5}=\frac{1}{G_{s}} \frac{L_{s}^{4}}{r_{2} r_{3} r_{4} r_{5}}
$$

and length parameter

$$
L_{D 5}=\sqrt{G_{s}} L_{s}
$$

Just as in the case of $p=1$ and $p=3$ we are free to set $K=0$. For $\overline{\mathrm{IIB}}$, this implies that one can take the 'OD1' limit even in the absence of NS5-branes. This yields a theory of D1-branes with strictly positive winding which is identical to the Wound D-string theory encountered in the previous subsection. That this theory is truly S-dual to the (IIB) Wound F-string theory is made explicit by the relation (68). The $K=0$ state in this theory does not have an open D-string sector - the role of these open strings in the theory is merely to describe excitations of NS5-branes when they happen to be present. It should also be noted that a generalization of the decoupling argument of Section 1 implies that the presence of a near-critical $C_{01^{-}}$ field is not strictly necessary in the Wound D-string (OD1) limit, although of course this field is a useful tool to implement the energy subtraction. It can be seen from Fig. 3 that the Wound D-string theory is $\mathrm{T}_{1}$-dual to DLCQ M-theory. This relation is just the $\mathrm{S}$-dual image of the Wound IIB $\leftrightarrow$ DLCQ IIA $\mathrm{T}_{1}$-duality. The mapping between the two theories converts D-string winding into longitudinal momentum, and NS5-branes into M5-branes.

Turning to $\widehat{\mathrm{IIB}}$ with $K=0$, we infer that IIB string theory on a five-torus has a limit (the 'OD5' limit) in which the basic degrees of freedom are wrapped D5-branes with strictly positive wrapping number. Extending our earlier nomenclature, it is natural to refer to this as the Wrapped D5-brane theory.

Let us pause here to note that one can similarly define Wrapped $\mathrm{D} p$-brane theories for $p=2,3,4$, which are all related to one another and to the Wrapped D1/D5-brane theories by T-dualities along directions 2345. It should be clear that for each value of $p$, the $\mathrm{OD} p$ theory constructed in [6, 33] is simply a truncation of the Wrapped $\mathrm{D} p$-brane theory to the set of states containing NS5-branes which extend along the 'Wrapped' directions. The case $p=0$ is special: here the names 'OD0' or 'Wrapped' 
are evidently not appropriate. The restriction that the 'Wrapped' limit induces in this case is simply that the D0-brane charge of all states be strictly positive, so as pointed out in [6] this is nothing but the original Matrix description of DLCQ Mtheory [27, 28]. We had already noted above that this theory is $\mathrm{T}_{1}$-dual to the Wound D-string theory.

Let us now return to the $p=5$ case. Since the $\widehat{\mathrm{IIB}}$ coupling $(70)$ diverges, Seiberg's argument [29, 30] for this case requires a final S-duality transformation (not shown in the figure) to obtain a weakly-coupled IIB' theory with

$$
g_{s}^{\prime}=G_{s} \frac{r_{2} r_{3} r_{4} r_{5}}{L_{s}^{4}} \epsilon^{1 / 2} \rightarrow 0, \quad l_{s}^{\prime}=\frac{L_{s}^{3}}{\sqrt{r_{2} r_{3} r_{4} r_{5}}}, \quad R_{1}^{\prime}=R_{1}, \quad R_{i}^{\prime}=\frac{G_{s} L_{s}^{2}}{r_{i}}
$$

with $K$ D5-branes and $N$ NS5-branes. The scaling $g_{s}^{\prime} \rightarrow 0$ at fixed $l_{s}^{\prime}$ would appear to yield the decoupled $(1,1)$ Little String Theory (LST) on the NS5-brane worldvolume [55]. However, as has been emphasized in [6], for $K \neq 0$ the presence of the D5branes implies that the IIB $^{\prime}$ is in fact strongly-coupled. There is no such obstruction in the case $K=0$, so we conclude that Wound IIB theory with no D5-branes is $\left(\mathrm{S} \cdot \mathrm{T}_{2345} \cdot \mathrm{S}\right)$-dual to $(1,1) \mathrm{LST}$.

\section{$5.3 p=2$}

As indicated in Fig. 4, Wound IIA on $\mathbf{T}^{2}$ can be lifted to M-theory on $\mathbf{T}^{3}$, with parameters

$$
l_{P}=G_{s}^{1 / 3} L_{s} \epsilon^{1 / 3} \rightarrow 0, \quad R_{1}=R_{1}, \quad R_{2}=r_{2} \sqrt{\epsilon} \rightarrow 0, \quad R_{10}=G_{s} L_{s} .
$$

Under the lift, the $K$ Wound D2-branes and $N$ F-strings become an M2-brane bound state with wrapping number $N>0$ on the $x^{1}-x^{10}$ torus [6]. In other words, the Wound limit of IIA maps to a limit (74) of M-theory which involves singling out two compact directions (in this case 1 and 10) and scaling $l_{P} \rightarrow 0$ in such a way that only objects with strictly positive M2 wrapping number on the 1-10 torus remain in the spectrum. Leaving $K$ arbitrary means that M2-branes are free to also wrap around the $x^{2}$ circle; $K=0$ is the special case where the M2-branes extend solely along $x^{1}$ and $x^{10}$. Extending our previous terminology, it is appropriate to refer to this as the Wrapped M2-brane theory. We will return to it in the next subsection.

Regarding $x^{2}$ as the M-theory circle, one can descend (following the dotted arrow in Fig. 4) to a ten-dimensional theory denoted as $\widehat{\mathrm{IIA}}$ in Fig. 4, with

$$
\hat{g}_{s}=G_{s}^{-1 / 2}\left(\frac{r_{2}}{L_{s}}\right)^{3 / 2} \epsilon^{1 / 4} \rightarrow 0, \quad \hat{l_{s}}=\sqrt{\frac{G_{s} L_{s}^{3}}{r_{2}}} \epsilon^{1 / 4} \rightarrow 0, \quad \hat{R}_{1}=R_{1}, \quad \hat{R}_{2}=G_{s} L_{s}
$$

$N$ D2-branes, and $K$ units of F-string winding. This is $(2+1)$-dimensional $U(N)$ SYM with $K$ units of $F_{02}$-flux. This is the theory which Seiberg's argument [29, 30] puts forth as the non-perturbative (Matrix) description of the system in question 


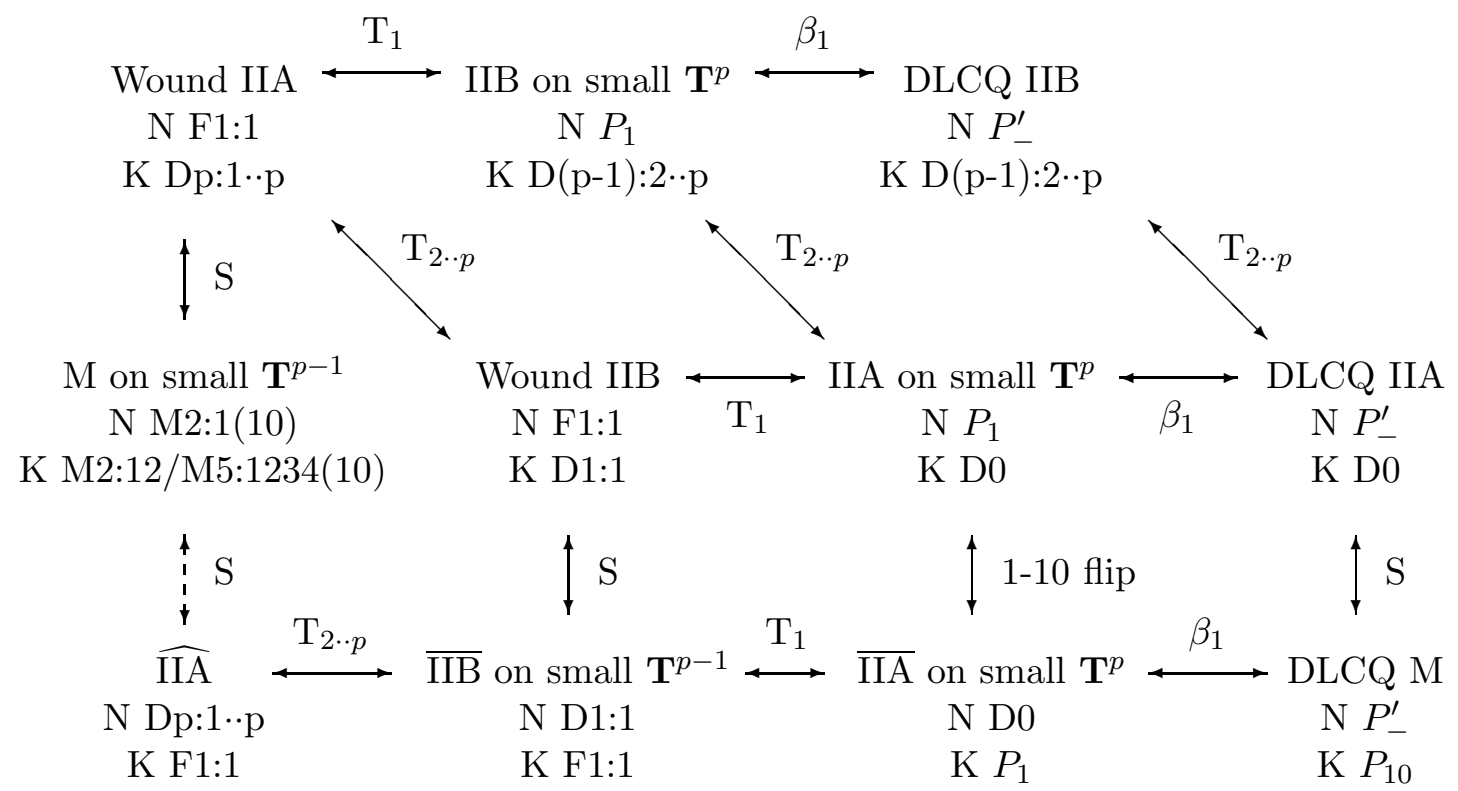

Figure 4: Same as Fig. 2, for $p=2$, 4. The two figures are related through transverse T-duality. Again, compact directions are not mentioned unless they have vanishing size in the relevant metric. Double entries for the object with multiplicity $K$ apply respectively to the $p=2,4$ cases. The dotted arrow connecting M-theory to $\widehat{\mathrm{IIA}}$ applies only in the $p=2$ case. The Wound IIA setup in the upper-left corner has hitherto been known as $p+1$ NCOS theory. Seiberg's derivation of a non-perturbative (Matrix) description of DLCQ M-theory on $\mathbf{T}^{p}$ proceeds along the bottom line of the figure.

[27, 54]. Its coupling constant is

$$
g_{Y M}^{2}=\frac{\hat{g}_{s}}{\hat{l}_{s}}=\frac{R_{1}^{2}}{L_{s}^{3}}
$$

It is again obvious from this perspective that one is free to set $K=0$. Just like in the $p=3$ case, it is curious to note that this is independent of the Wound coupling $G_{s}$, but again it should be emphasized that this does not imply the existence of some regime where the two descriptions can be examined perturbatively at the same time.

\section{$5.4 p=4$}

The direct lift of Wound IIA to eleven dimensions takes us to M-theory with parameters (74), $R_{3,4}=r_{3,4} \sqrt{\epsilon}$, in the presence of $N$ M2-branes and $K$ M5-branes. When bound to the fivebranes, the membranes appear as $N$ units of $H_{01(10)^{-}}$flux, where $H$ is the (self-dual) three-form field strength on the fivebrane worldvolume. This field (or equivalently, the bulk $A_{01(10)}$ gauge field) becomes critical in the Wound/DLCQ limit, yielding a framework known as OM theory [6, 31]. Reasoning like before, we see that the possibility of setting $K=0$ means that it is possible to define an 'OM' limit 
of M-theory even if there are no M5-branes present, to obtain a theory which contains M2-branes with strictly positive wrapping number on the $x^{1}-x^{10}$ torus. This is of course the Wrapped M2-brane theory discussed in the previous subsection. M5-branes remain in the spectrum of this theory only if they carry a positive M2 1-10 wrapping number. When they are present, their excitations include open M2-branes- this is the standard OM theory setup.

We should emphasize that, despite the fact that $l_{P} \rightarrow 0$, the rescaling of the transverse directions seen in (74) implies that the Wrapped M2 theory is not just the conformal $S O(8)$-invariant theory on the M2-brane worldvolume - rather, it is a limit of the full eleven-dimensional M-theory. States in this theory can contain several interacting clumps of M2-branes with distinct (positive) 1-10 wrapping number, or even M5-branes, as we have seen above.

Following Seiberg's procedure [29, 30] one arrives in this case at the theory labelled $\widehat{\mathrm{IIA}}$ in Fig. 4, only to realize that its coupling constant diverges, signalling the need to employ an $\widehat{\mathrm{M}}$-theory description (not shown in the figure) with

$$
\hat{l_{P}}=G_{s}^{2 / 3} \frac{L_{s}^{2}}{\left(r_{2} r_{3} r_{4}\right)^{1 / 3}} \epsilon^{1 / 6} \rightarrow 0, \quad \hat{R}_{1}=R_{1}, \quad \hat{R}_{i}=G_{s} \frac{L_{s}^{2}}{r_{j} r_{k}}, \quad \hat{R}_{10}=G_{s} L_{s} .
$$

In the third equation $i, j, k$ are understood to take different values, so for example $\hat{R}_{2}=G_{s} L_{s}^{2} / r_{3} r_{4}$. In this picture there are $N$ M5-branes and $K$ M2-branes present, and the limit $l_{P} \rightarrow 0$ yields the decoupled $(2,0)$ theory on the M5-brane worldvolume. This is the theory conjectured to capture the physics of DLCQ M-theory on $\mathbf{T}^{4}$ at the non-perturbative level [56, 57]. The $K$ M2-branes are realized as $H_{01(10)}$-flux in the $(2,0)$ theory, and as in all other cases it is clear that one is free to set $K=0$.

\section{Conclusions}

In this paper we have analyzed the T-duality relation between the DLCQ limit of IIA/B theories and the NCOS theories [4, 5, 12]. In the process, we have discovered that it is meaningful to consider an 'NCOS' limit of the IIA/B theory without branes. We compactify the IIA/B theory on a circle of radius $R$ and consider a sector with definite, conserved, total winding number $W$. The low energy limit $l_{s} \rightarrow 0$ can be taken keeping $g_{s} l_{s}$ and $R$ fixed, and turns out to be exactly T-dual to the limit that defines the DLCQ IIB/A with $W$ units of momentum along the lightlike direction. One finds that the energy of a string with winding number $w$ has a contribution $|w| R / l_{s}^{2}$ which diverges in the limit. If we restrict attention to the subsector where all winding numbers are strictly positive $(|w|=w>0)$, this divergent contribution can be subtracted, since the total winding $W=\sum w_{i}=\sum\left|w_{i}\right|$ is conserved in all interactions. As we have discussed in Section 3, the subtraction is equivalent to considering the theory in the presence of a critical $B_{01}$-field, but since this field can be gauged away the physics cannot change. The T-dual procedure in the DLCQ limit is to boost along the circle, which also does not change the physics. The rules of T-duality show that boosting and turning on a $B_{01}$-field are precisely equivalent. 
We call the resulting theory the Wound IIA/B theory, since it is characterized by the fact that all objects in it carry strictly positive F-string winding. At low energy the loop-counting parameter in the theory is $g_{s} l_{s} / R$, as we have checked in Section 4.2 by means of a one-loop calculation. However, since in the IIB case the Wound theory is in a certain sense (see below) S-dual to $1+1 U(W)$ SYM with $g_{Y M} \sim 1 /\left(g_{s} l_{s}\right)$ [6, 12], which is strongly coupled in the infrared, we expect the effective coupling at energies $E \gg 1 / R$ to grow as $g_{s} l_{s} E$. For these energies the length scale $g_{s} l_{s}$ is the meaningful parameter of the theory. The scattering amplitudes in the Wound IIA/B theory are similar to those in the usual string theory, even though the momentum energy relation is non-relativistic with mass and energy separately conserved (in this context winding number is interpreted as a mass). This is clear since the theory is perturbatively equivalent (T-dual) to the standard DLCQ, replacing winding number by momentum in the compact direction.

In Section 4.3 we have considered the Wound theory in the presence of D-branes. We have seen there that in order for a brane to remain in the spectrum, it must carry an electric flux along the Wound circle, or, equivalently, a strictly positive fundamental-string winding number. In that case the limit for the theory on the brane reduces to the corresponding NCOS theory, and the Wound theory emerges as the framework that unifies these setups, which up to now had been regarded as isolated theories. T-duality implies that NCOS theory is also the appropriate way to introduce D-branes in DLCQ IIA. For example, as shown in Section 2.2, the noncommutativity in the DLCQ picture arises from the fact that left- and right-moving momenta are different, which, as a consequence, gives a phase when two open string vertices are interchanged. In the decompactification limit $R \rightarrow \infty$ the NCOS theories are obtained as decoupled theories on the branes.

In Section 5 we studied the Wound theory compactified on transverse tori. By use of diverse dualities, we were naturally led to various Wrapped $p$-Brane theories, which are obtained as limits of IIA/B/M-theory that leave in the spectrum only those objects which carry strictly positive brane wrapping number on a specific $p$-torus. Just like the Wound F-string theory unifies the various NCOS setups, these Wrapped Brane theories contain the extensions of NCOS theories to open $p$-brane theories, i.e., OM theory [6, 31] and $\mathrm{OD} p$ theories [33, 6]. These theories have already appeared in the process of deriving Matrix models for DLCQ IIA/M-theory on a transverse torus [29, 30], but our improved understanding allows us to consider them as theories in their own right.

As discussed in Section 5.3, as long as there are (at least) two compact dimensions, it is possible to take a limit of M-theory which truncates the spectrum down to those objects carrying strictly positive M2 wrapping number. This is the Wrapped M2brane theory, which includes OM theory as a particular set of states - namely, those that contain M5-branes extended along the 'wrapped' directions. This M-theoretic construct is related to similar string-theoretic frameworks in the usual manner. If we start with the Wrapped M2-brane theory and descend to ten dimensions by identifying one of the 'Wrapped' directions as the M-theory circle, we obtain the Wound IIA 
theory. If, on the other hand, the M-theory circle is transverse to the directions of M2 wrapping, the result is the Wrapped D2-brane theory. Using transverse T-duality one can then reach the Wound IIB theory, and all the other Wrapped Dp-brane theories. Additionally, starting with the Wound (IIB) F-string and D-string theories, longitudinal T-duality brings us to DLCQ IIA and DLCQ M-theory, respectively. Finally, one is led to propose the existence of the analogous theories of Wrapped NS5-branes and M5-branes when seeking S-dual descriptions for all of the Wrapped brane theories encountered so far.

We would like to emphasize that the Wrapped point of view is distinct from that of Matrix theory [27, 28]. When attempting to provide a non-perturbative formulation of DLCQ IIA/M-theory on transverse tori of different dimensions, the Matrix approach focuses attention on models which are defined on spacetimes of different dimensionality [29, 30]. The Wrapped $p$-brane theories, on the other hand, are always defined on a ten- or eleven-dimensional spacetime (with at least $p$ compact directions).

To make this difference clearer, let us pose a specific question: what is the S-dual of the Wound IIB theory? Since Wound IIB is defined as IIB in the limit (38), the answer is clear: S-dualizing one obtains a ten-dimensional theory which is defined as IIB in the limit

$$
\delta \rightarrow 0, \quad g_{s}=\frac{1}{G_{s}} \sqrt{\delta}, \quad l_{s}=\sqrt{G_{s}} L_{s} \delta^{1 / 4}, \quad h=\delta, \quad \text { with } \quad G_{s}, L_{s}, R \quad \text { fixed } .
$$

This is true independently of whether the Wound theory is compactified on a transverse torus or not. Since the requirement that objects in the Wound theory carry strictly positive F-string winding is mapped to the condition that objects in the Sdual theory carry strictly positive D-string winding, it is reasonable to call this the Wound D-string theory. The relation to the Matrix approach is that, in the absence of a transverse compactification, the limit (78) reduces the theory on the D1-brane worldvolume to $1+1$ SYM (i.e., Matrix String theory). If we disregard branes of infinite extent, then with only one compact direction there are no other possible branes in the Wound D-string theory ${ }^{13}$, so it can be effectively identified with $1+1$ SYM.

Suppose now that we compactify the Wound IIB theory on, say, a (transverse) two-torus. The S-dual Wound D-string theory can then have D3-branes wrapped on the resulting (transverse + longitudinal) three-torus, as long as they carry positive D-string winding. This means there is a magnetic flux on the D3-brane worldvolume, and as noted in [5] and in Section 5.1] of the present paper, the limit (78) yields the $(3+1)$-dimensional NCYM theory. Similarly, if we compactify on a transverse four-torus, there can be NS5-branes carrying D-string winding number, and the limit (78) defines what has been called the OD1 theory [33, 6] (open D-strings describe excitations of the NS5-brane). The role of the Wound D-string theory as a unifying framework which can accommodate all of these different degrees of freedom is thus clear. It is also evident that $1+1 \mathrm{SYM}$ cannot play this same role (at least not

\footnotetext{
${ }^{13}$ Except of course F-strings with adsorbed D-strings, which are easily accommodated in the $1+1$ SYM description [12].
} 
directly, and for finite $N$ ). The reason is that in the SYM description, the radii of the transverse circles are shrinking to zero size, due to the scaling of the transverse metric parameter $h$ built into (78). The Matrix approach [29, 30] therefore considers this description useless, and carries out further dualities to arrive at $(3+1)$-dimensional SYM and $(5+1)$-dimensional $(1,1)$ LST, respectively.

Summarizing, the NCOS theories describe branes in the Wound IIB/A theory T-dual to the full DLCQ IIA/B theory. Similarly, OM theory and the ODp theories describe M5-branes and NS5-branes in the Wrapped M2 and Wrapped Dp-brane theories - theories which are U-dual to Wound IIA/B. It would be worthwhile to explore the possible additional implications that this unified perspective could have for the theories involved. In particular, the NCOS theories on a circle provide a simple way to capture the physics of D-branes in the DLCQ limit of string theory: through T-duality each brane is described by a non-commutative open string theory. It would be interesting to use this correspondence to try to gain a better understanding of the physics of DLCQ.

Note Added: While this paper was being written, the work [58] appeared, which overlaps with our Sections 2.2 and 5 .

\section{Acknowledgements}

AG would like to thank Ansar Fayyazuddin, Bo Sundborg, and especially Subir Mukhopadhyay for valuable conversations. We are grateful to F. Kristianson and P. Rajan for useful discussions. The work of UD and AG was supported by the Swedish Natural Science Research Council (NFR).

\section{References}

[1] A. Connes, M. R. Douglas and A. Schwarz, "Noncommutative geometry and matrix theory: Compactification on tori," JHEP 9802, 003 (1998), hep-th/9711162.

[2] M. R. Douglas and C. Hull, "D-branes and the noncommutative torus," JHEP 9802, 008 (1998), hep-th/9711165.

[3] N. Seiberg and E. Witten, "String theory and noncommutative geometry," JHEP 9909, 032 (1999), hep-th/9908142.

[4] N. Seiberg, L. Susskind and N. Toumbas, "Strings in background electric field, space/time non-commutativity and a new noncritical string theory," JHEP 0006, 021 (2000), hep-th/0005040. 
[5] R. Gopakumar, J. Maldacena, S. Minwalla and A. Strominger, "S-duality and noncommutative gauge theory," JHEP 0006, 036 (2000), hep-th/0005048.

[6] R. Gopakumar, S. Minwalla, N. Seiberg and A. Strominger, "OM theory in diverse dimensions," hep-th/0006062.

[7] O. Aharony, J. Gomis and T. Mehen, "On theories with light-like noncommutativity," JHEP 0009, 023 (2000) hep-th//0006236.

[8] N. Seiberg, L. Susskind and N. Toumbas, "Space/time non-commutativity and causality," JHEP 0006, 044 (2000), hep-th/0005015.

[9] J. L. Barbon and E. Rabinovici, "Stringy fuzziness as the custodian of time-space noncommutativity," Phys. Lett. B486, 202 (2000), hep-th/0005073.

[10] J. Gomis and T. Mehen, "Space-time noncommutative field theories and unitarity," hep-th/0005129.

[11] O. J. Ganor, G. Rajesh and S. Sethi, "Duality and non-commutative gauge theory," hep-th/0005046.

[12] I. R. Klebanov and J. Maldacena, " $1+1$ dimensional NCOS and its U(N) gauge theory dual," hep-th/0006085.

[13] C. P. Herzog and I. R. Klebanov, "Stable massive states in $1+1$ dimensional NCOS," hep-th/0009017.

[14] T. Harmark, "Supergravity and space-time non-commutative open string theory," JHEP 0007, 043 (2000), hep-th/0006023.

[15] V. Sahakian, "The phases of 2-D NCOS," JHEP 0009, 025 (2000) hep-th/0008073.

[16] S. S. Gubser, S. Gukov, I. R. Klebanov, M. Rangamani and E. Witten, "The Hagedorn transition in non-commutative open string theory," hep-th/0009140.

[17] S. Gukov, I. R. Klebanov and A. M. Polyakov, "Dynamics of (n,1) strings," Phys. Lett. B423, 64 (1998), hep-th/9711112.

[18] I. R. Klebanov, " $1+1$ Dimensional NCOS and its $U(N)$ Gauge Theory Dual," Talk at Strings 2000, University of Michigan (July 13, 2000), http://feynman.physics.lsa.umich.edu/cgi-bin/s2ktalk.cgi?klebanov .

[19] J. Maharana and S. S. Pal, "Noncommutative open string, D-brane and duality," Phys. Lett. B488, 410 (2000), hep-th/0005113.

[20] G. Chen and Y. Wu, "Comments on noncommutative open string theory: Vduality and holography," hep-th/0006013. 
[21] J. X. Lu, S. Roy and H. Singh, "((F,D1),D3) bound state, S-duality and noncommutative open string / Yang-Mills theory," JHEP 0009, 020 (2000), hep-th/0006193.

[22] J. G. Russo and M. M. Sheikh-Jabbari, "On noncommutative open string theories," JHEP 0007, 052 (2000), hep-th/0006202.

[23] S. Rey and R. von Unge, "S-duality, noncritical open string and noncommutative gauge theory," hep-th/0007089.

[24] R. Cai and N. Ohta, "(F1, D1, D3) bound state, its scaling limits and SL(2,Z) duality," hep-th/0007106.

[25] J. X. Lu, S. Roy and H. Singh, "SL(2,Z) duality and 4-dimensional noncommutative theories," hep-th/0007168.

[26] M. Gremm, "Compactified NCOS and duality," hep-th/0009095.

[27] T. Banks, W. Fischler, S. H. Shenker and L. Susskind, "M theory as a matrix model: A conjecture," Phys. Rev. D55, 5112 (1997), hep-th/9610043.

[28] L. Susskind, "Another conjecture about M(atrix) theory," hep-th/9704080.

[29] N. Seiberg, "Why is the matrix model correct?," Phys. Rev. Lett. 79, 3577 (1997), hep-th/9710009.

[30] A. Sen, "D0 branes on $T^{n}$ and matrix theory," Adv. Theor. Math. Phys. 2, 51 (1998), hep-th/9709220.

[31] E. Bergshoeff, D. S. Berman, J. P. van der Schaar and P. Sundell, "Critical fields on the M5-brane and noncommutative open strings," hep-th/0006112.

[32] E. Bergshoeff, D. S. Berman, J. P. van der Schaar and P. Sundell, "A noncommutative M-theory five-brane," hep-th/0005026.

[33] T. Harmark, "Open branes in space-time non-commutative little string theory," hep-th/0007147.

[34] T. Kawano and S. Terashima, "S-duality from OM-theory," hep-th/0006225.

[35] C. G. Callan and I. R. Klebanov, "D-Brane Boundary State Dynamics," Nucl. Phys. B465, 473 (1996), hep-th/9511173.

[36] H. Verlinde, "A matrix string interpretation of the large N loop equation," hep-th/9705029.

[37] L. Motl, "Proposals on nonperturbative superstring interactions," hep-th/9701025. 
[38] T. Banks and N. Seiberg, "Strings from matrices," Nucl. Phys. B497, 41 (1997), hep-th/9702187.

[39] R. Dijkgraaf, E. Verlinde and H. Verlinde, "Matrix string theory," Nucl. Phys. B500, 43 (1997), hep-th/9703030.

[40] J. Maldacena, "The Large $N$ Limit of Superconformal Field Theories and Supergravity," Adv. Theor. Math. Phys. 2 (1998) 231, hep-th/9711200.

[41] J. Polchinski, String Theory, Cambridge University Press (1998), Vol. 1.

[42] V. Balasubramanian, R. Gopakumar and F. Larsen, "Gauge theory, geometry and the large N limit," Nucl. Phys. B526, 415 (1998), hep-th/9712077.

[43] A. Güijosa, "Is physics in the infinite momentum frame independent of the compactification radius?," Nucl. Phys. B533, 406 (1998), hep-th/9804034.

[44] S. Hellerman and J. Polchinski, "Compactification in the lightlike limit," Phys. Rev. D59, 125002 (1999), hep-th/9711037.

[45] A. Bilal, "A comment on compactification of M-theory on an (almost) light-like circle," Nucl. Phys. B521, 202 (1998) hep-th/9801047].

[46] A. Bilal, "DLCQ of M-theory as the light-like limit," Phys. Lett. B435, 312 (1998), hep-th/9805070.

[47] S. Uehara and S. Yamada, "On the DLCQ as a light-like limit in string theory," hep-th/0008146.

[48] A. Giveon, M. Porrati and E. Rabinovici, "Target space duality in string theory," Phys. Rept. 244, 77 (1994), hep-th/9401139.

[49] S. Weinberg, "Dynamics At Infinite Momentum," Phys. Rev. 150, 1313 (1966).

[50] J. Kogut and L. Susskind, "The Parton Picture Of Elementary Particles," Phys. Rept. 8, 75 (1973).

[51] T. Maskawa and K. Yamawaki, "The Problem Of P $+=$ O Mode In The Null Plane Field Theory And Dirac's Method Of Quantization," Prog. Theor. Phys. 56, 270 (1976).

[52] H. C. Pauli and S. J. Brodsky, "Discretized Light Cone Quantization: Solution To A Field Theory In One Space One Time Dimensions," Phys. Rev. D32, 2001 (1985).

[53] J. A. Harvey, "Spin dependence of D0-brane interactions," Nucl. Phys. Proc. Suppl. 68, 113 (1998), hep-th/9706039. 
[54] W. I. Taylor, "D-brane field theory on compact spaces," Phys. Lett. B394, 283 (1997), hep-th/9611042.

[55] N. Seiberg, "New theories in six dimensions and matrix description of M-theory on $\mathbf{T}^{5}$ and $\mathbf{T}^{5} / \mathbf{Z}_{2}$," Phys. Lett. B408, 98 (1997), hep-th/9705221.

[56] M. Rozali, "Matrix theory and U-duality in seven dimensions," Phys. Lett. B400, 260 (1997), hep-th/9702136.

[57] M. Berkooz, M. Rozali and N. Seiberg, "Matrix Description of M-theory on $\mathbf{T}^{4}$ and $\mathbf{T}^{5}$," Phys. Lett. B408, 105 (1997), hep-th/9704089.

[58] S. Hyun, "U-duality between NCOS theory and matrix theory," hep-th/0008213. 